\title{
Mitochondrial dysfunction enhances cisplatin resistance in human gastric cancer cells via the ROS-activated GCN2-eIF2a- ATF4-XCT pathway
}

\author{
Sheng-Fan Wang ${ }^{1,2}$, Meng-Shian Chen ${ }^{1}$, Yueh-Ching Chou ${ }^{1,2,3}$, Yune-Fang Ueng ${ }^{1,4,5}$, \\ Pen-Hui Yin ${ }^{6}$, Tien-Shun Yeh${ }^{7}$, Hsin-Chen Lee ${ }^{1}$ \\ ${ }^{1}$ Department and Institute of Pharmacology, School of Medicine, National Yang-Ming University, Taipei, Taiwan \\ ${ }^{2}$ Department of Pharmacy, Taipei Veterans General Hospital, Taipei, Taiwan \\ ${ }^{3}$ School of Pharmacy, Taipei Medical University, Taipei, Taiwan \\ ${ }^{4}$ National Research Institute of Chinese Medicine, Ministry of Health and Welfare, Taipei, Taiwan \\ ${ }^{5}$ Institute of Medical Sciences, Taipei Medical University, Taipei, Taiwan \\ ${ }^{6}$ Department of Medical Research, Taipei Veterans General Hospital, Taipei, Taiwan \\ ${ }^{7}$ Institute of Anatomy and Cell Biology, School of Medicine, National Yang-Ming University, Taipei, Taiwan \\ Correspondence to: Hsin-Chen Lee, email: hclee2@ym.edu.tw \\ Keywords: gastric cancer, mitochondrial dysfunction, cisplatin resistance, $x C T$, retrograde signaling \\ Received: June 14,2016 Accepted: September 21,2016 Published: September 30, 2016
}

\section{ABSTRACT}

Mitochondrial DNA mutations and defects in mitochondrial enzymes have been identified in gastric cancers, and they might contribute to cancer progression. In previous studies, mitochondrial dysfunction was induced by oligomycin-enhanced chemoresistance to cisplatin. Herein, we dissected the regulatory mechanism for mitochondrial dysfunction-enhanced cisplatin resistance in human gastric cancer cells. Repeated cisplatin treatment-induced cisplatin-resistant cells exhibited high SLC7A11 (XCT) expression, and XCT inhibitors (sulfasalazine or erastin), XCT siRNA, or a GSH synthesis inhibitor (buthionine sulphoximine, BSO) could sensitize these cells to cisplatin. Clinically, the high expression of XCT was associated with a poorer prognosis for gastric cancer patients under adjuvant chemotherapy. Moreover, we found that mitochondrial dysfunction enhanced cisplatin resistance and up-regulated XCT expression, as well as intracellular glutathione (GSH). The xCT inhibitors, siRNA against XCT or BSO decreased mitochondrial dysfunction-enhanced cisplatin resistance. We further demonstrated that the upregulation of the eIF2a-ATF4 pathway contributed to mitochondrial dysfunction-induced XCT expression, and activated eIF $2 a$ kinase GCN2, but not PERK, stimulated the eIF2a-ATF4-XCT pathway in response to mitochondrial dysfunction-increased reactive oxygen species (ROS) levels. In conclusion, our results suggested that the ROS-activated GCN2-eIF2a-ATF4-xCT pathway might contribute to mitochondrial dysfunction-enhanced cisplatin resistance and could be a potential target for gastric cancer therapy.

\section{INTRODUCTION}

Gastric cancer is one of the most common cancers worldwide. The incidence rate of gastric cancer was highest in East Asia, Eastern Europe, and South America [1]. The main treatment modality for gastric cancer is surgery, except for in the advanced or unresectable stages. Systemic chemotherapy based on 5-fluorouracil (5-FU) plus an anthracycline agent or cisplatin-containing combinations, is the most effective treatment modality for patients with metastatic disease [2]. However, chemotherapy resistance remains an important issue in the treatment of advanced gastric cancer patients.

Cancer cells usually exhibit an aberrant metabolism, and deregulated cellular energetics were recently included among the hallmarks of cancer [3]. Mitochondria are intracellular organelles that participate in bioenergetics metabolism and cellular homeostasis, including the 
generation of adenosine triphosphate (ATP) through oxidative phosphorylation, intermediate metabolism, the production of reactive oxygen species (ROS), and the regulation of apoptosis [4]. In past decades, somatic mutations in the mitochondrial genome have been found to be frequent events in human gastric cancers, and most of these mitochondrial DNA (mtDNA) mutations can lead to mitochondrial dysfunction [5,6]. Mitochondrial dysfunction has been suggested to contribute to cancer progression $[7,8]$.

Accumulating evidence has revealed that the mitochondria can generate mitochondria-to-nucleus (retrograde) signals to regulate cellular function and to protect against mitochondrial dysfunction by activating the expression of nuclear genes involved in metabolic reprogramming or stress defense [7]. The retrograde signaling pathways have thus been proposed to be involved in mitochondrial dysfunction-enhanced malignant progression [8]. In our previous study, mitochondrial dysfunction was found to promote a migration phenotype and cisplatin resistance in human gastric cancer cells [6]. Recently, various retrograde signaling pathways have been shown to contribute to mitochondrial dysfunction-enhanced cancer migratory/invasive abilities $[9,10]$. However, the molecular mechanism underlying mitochondrial dysfunction-induced cisplatin resistance remains unclear.

Several mechanisms can result in cisplatin resistance, including reduced intracellular drug accumulation, increased inactivation by thiol-containing molecules, e.g. glutathione (GSH), increased DNA damage repair, and the inhibition of apoptosis [11]. Among these mechanisms, increases in GSH have been demonstrated in a number of cisplatin-resistant tumor models and have been confirmed in clinical studies [11]. Moreover, elevated GSH might increase DNA repair or increase the inhibitory effect on apoptosis by buffering drug-induced oxidative stress [12]. An increased intracellular GSH level is thus generally accepted to be a significant mechanism for cisplatin resistance.

The system $\mathrm{x}_{\mathrm{c}}^{-}$cystine/glutamate antiporter is a plasma membrane transporter mediating the cellular uptake of cystine from the microenvironment in exchange for intracellular glutamate [13]. This transporter is known to contribute to the maintenance of intracellular GSH levels and to protect cells from oxidative stress or toxic compounds [13]. Human system $\mathrm{x}_{\mathrm{c}}^{-}$cystine/glutamate antiporter consists of the heavy subunit $4 \mathrm{~F} 2 \mathrm{hc}$ (found in a variety of amino-acid transporters) and the light subunit $\mathrm{xCT}$ (SLC7A11). The transport activity is thus believed to be determined by $\mathrm{xCT}$ [13]. It was reported that $\mathrm{xCT}$ gene expression could be up-regulated by nuclear factor erythroid 2-related factor-2 (Nrf2), which binds to antioxidant response elements (AREs) [14], or by the activating transcription factor 4 (ATF4), which binds to amino acid response elements (AAREs) in the $\mathrm{xCT}$ promoter $[15,16]$.
The eukaryotic initiation factor $2 \alpha$ (eIF2 $\alpha$ )-ATF4 pathway is the central regulator of the integrated stress response. The phosphorylation of Ser-51 of the eukaryotic initiation factor eIF $2 \alpha$ attenuates the initiation of global cap-dependent protein translation, but it concurrently increases the cap-independent translation of select mRNAs with small upstream open reading frames, including the ATF4 transcription factor [17]. The increase in eIF $2 \alpha$ phosphorylation leads to the adaptation of the cell to stress conditions or, alternatively, to the induction of apoptosis $[17,18]$. Moreover, four eIF2 $\alpha$ kinases have been identified in mammals, and dsRNA-activated protein kinase R (PKR), heme-regulated inhibitor eIF2 $\alpha$ kinase (HRI), protein kinase R-like endoplasmic reticulum kinase (PERK), and general control nonderepressible-2 (GCN2) are activated by distinct stresses [17]. Recent reports have suggested that GCN2 or PERK might contribute to increased eIF $2 \alpha$ phosphorylation during different types of mitochondrial stress $[19,20]$. The activation of the eIF2 $\alpha$ ATF4 pathway and the eIF $2 \alpha$ kinases might be involved in retrograde signaling pathways.

In this study, we evaluated the role of $\mathrm{xCT}$ in the mitochondrial dysfunction-enhanced cisplatin resistance of human gastric cancer cells. Cisplatin-resistant gastric cancer cells were established to validate high $\mathrm{xCT}$ expression in cisplatin resistance. Moreover, the molecular mechanisms involved in the eIF2 $\alpha$-ATF4 pathway and the eIF $2 \alpha$ kinases were investigated.

\section{RESULTS}

\section{Cisplatin-resistant gastric cancer cells have high xCT expression and rely on environmental cystine for cell survival}

To establish cisplatin-resistant gastric cancer cells, we repeatedly treated gastric cancer cells with increasing concentrations of cisplatin, starting from a low dose. We obtained three lines of cisplatin-resistant gastric cancer cells derived from SC-M1, AGS, and AZ521 cells. Figure 1A shows that these cisplatin-resistant gastric cancer cells exhibited a significantly decreased sensitivity to cisplatin (IC50 of cisplatin: $2.23 \mu \mathrm{g} / \mathrm{ml}$ for SC-M1, $>25 \mu \mathrm{g} / \mathrm{ml}$ for SC-M1CisR; $2.31 \mu \mathrm{g} / \mathrm{ml}$ for AGS, $15.83 \mu \mathrm{g} / \mathrm{ml}$ for AGSCisR; $2.39 \mu \mathrm{g} / \mathrm{ml}$ for AZ521, $18.71 \mu \mathrm{g} / \mathrm{ml}$ for AZ521CisR). We found that the protein expression of multidrug-resistance protein (MDR) and the function of MDR1 (p-glycoprotein, P-gp) and multidrug resistance-associated protein (MRP) were not increased in the cisplatin-resistant cells, compared to the parental cells (Supplementary Figures S1A-S1C). Moreover, these cisplatin-resistant cancer cells had higher $\mathrm{xCT}$ gene and protein expression (Figures 1B and 1C). In addition, higher intracellular GSH levels (Figure 1D) were observed, compared with their parental SC-M1 cells. We also found that the cisplatin-resistant cells were more sensitive to 

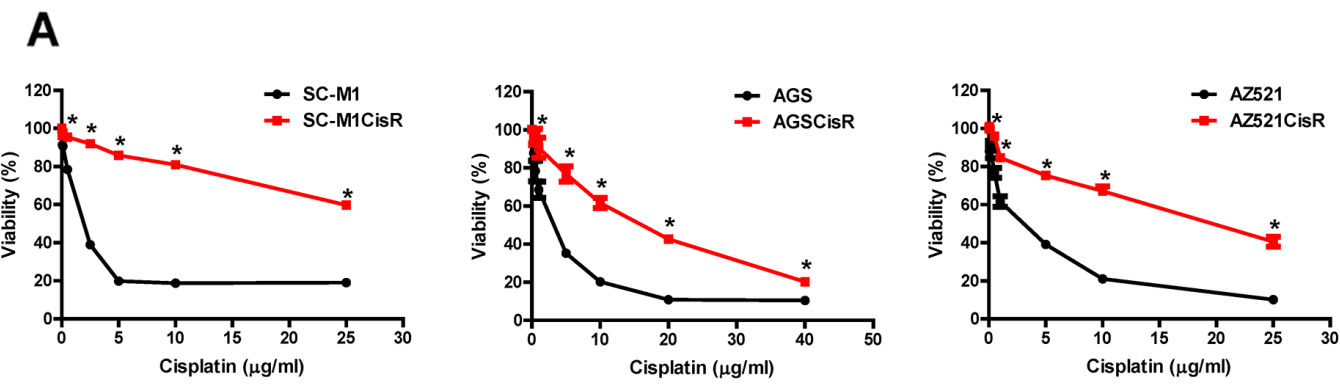

B

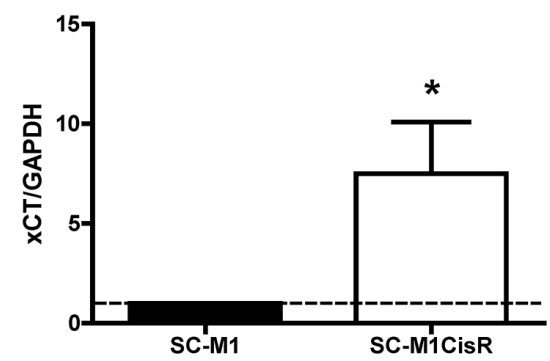

D

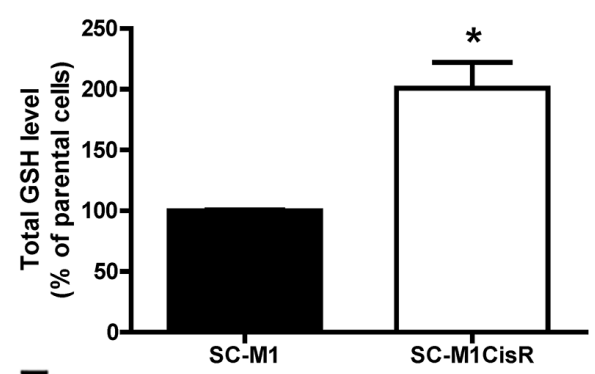

$\mathbf{F}$

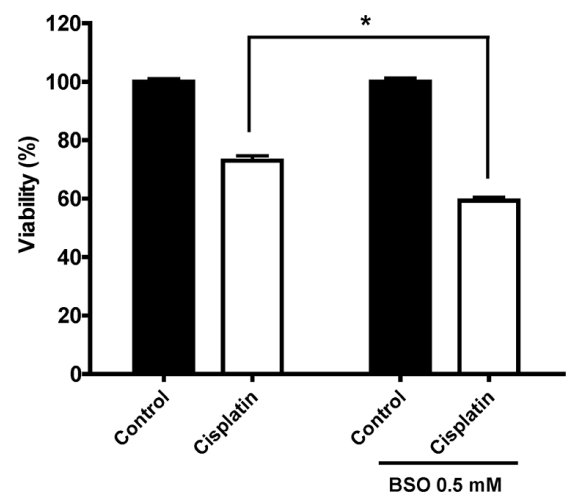

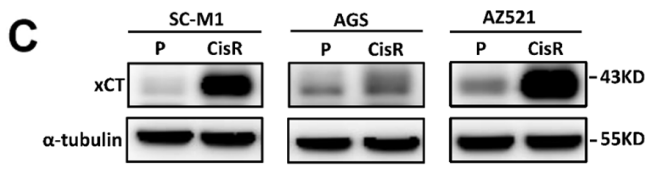

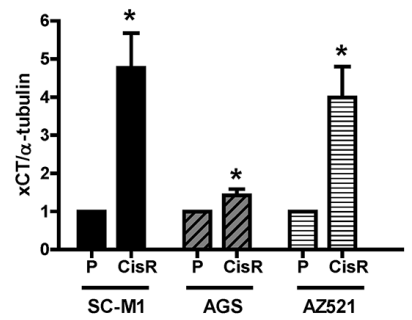

E

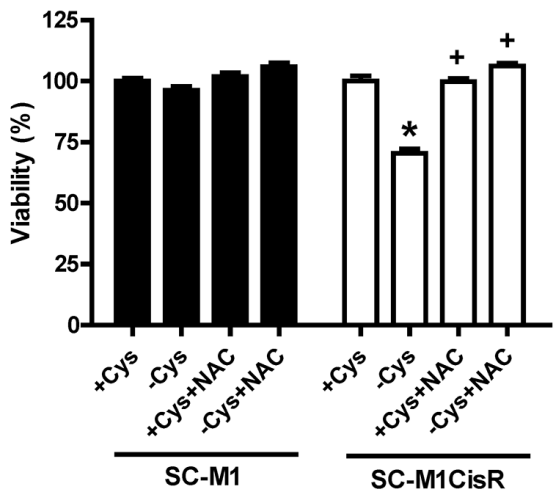

Figure 1: Cisplatin-resistant gastric cancer cells have high $\mathrm{xCT}$ expression and rely on environmental cystine for cell survival. A. The cisplatin-resistant gastric cancer cells (cisplatin-resistant, CisR) and their parental SC-M1, AGS, and AZ521 cells were treated with cisplatin for $48 \mathrm{~h}$. The sensitivity was determined by SRB assay. B. qRT-PCR analysis of xCT mRNA between the SC-M1 and the cisplatin-resistant SC-M1 gastric cancer cells. qRT-PCR values were normalized to GAPDH mRNA. C. Western blot analysis of xCT protein expression between the parental and the cisplatin-resistant gastric cancer cells. D. The parental SC-M1 and cisplatin-resistant SCM1 cells (SC-M1CisR) were seeded at a density of $1 \times 10^{6}$ cells per 10-cm dish. After culture overnight, the cells were collected and the total cellular GSH levels were analyzed by GSH assay kit. E. The SC-M1 parental and cisplatin-resistant (SC-M1CisR) gastric cancer cells were seeded at a density of 3000 cells per well in 96-well plates and were cultured in cystine-free medium (-Cys) and cystine-containing complete medium (+Cys). The cells were pre-treated with N-acetyl cysteine (NAC, $5 \mathrm{mM}$ ) for $12 \mathrm{~h}$, followed by co-treatment with cystinefree medium and cystine-containing complete medium for $72 \mathrm{~h}$. F. The SC-M1CisR cells were treated with BSO and/or cisplatin $(25 \mu \mathrm{g} /$ $\mathrm{ml}$ ) for $48 \mathrm{~h}$. The cell viability was determined by SRB assay. Data represent the mean \pm SEM of three independent experiments. ${ }^{*} p<0.05$, compared to the parental cells or + Cys group; $+p<0.05$, compared to the -Cys group. 
cystine-depletion medium than the parental cells, and $\mathrm{N}$-acetyl cysteine (NAC) reversed cystine depletioninduced cell death (Figure 1E). Moreover, buthionine sulphoximine (BSO, a GSH biosynthesis inhibitor) treatments increased the sensitivity of the cisplatinresistant cells to cisplatin (Figure 1F). These results suggested that $\mathrm{xCT}$-dependent GSH elevation might play an important role in the cell survival of cisplatin-resistant gastric cancer cells. Moreover, the basal ROS level in the cisplatin-resistant gastric cancer cells was lower than in the parental cells (Supplementary Figures S1D and S1E). Cisplatin resistance and cell survival might be ROSdependent in cisplatin-resistant gastric cancer cells.

\section{Inhibition and knockdown of $\mathrm{xCT}$ increase the cisplatin sensitivity of the cisplatin-resistant gastric cancer cells, and high $\mathrm{xCT}$ expression is a poor prognostic factor in gastric cancer patients under adjuvant chemotherapy treatment}

To evaluate further whether high $\mathrm{xCT}$ expression is essential for cisplatin resistance, we treated the cisplatinresistant cells with $\mathrm{xCT}$ inhibitors, such as sulfasalazine (SSA) and erastin. The results revealed that SSA and erastin partially increased the cell's sensitivity to cisplatin (Figures 2A and 2B). In addition, the knockdown of $\mathrm{xCT}$ expression by siRNA increased the cell's cisplatin sensitivity (Figures 2C and 2D). These results suggested that increased $\mathrm{xCT}$ expression could contribute to cisplatin resistance in human gastric cancer cells.

To understand the clinical impacts of $\mathrm{xCT}$ expression in gastric cancer patients, we used an online-database (http://kmplot.com/analysis/) and analyzed the effect of xCT expression on overall survival (OS) and progression-free survival (PFS) in gastric cancer patients with chemotherapy treatment. In gastric cancer patients within the clinical cohort undergoing adjuvant chemotherapy $(\mathrm{n}=153)$, we found that high $\mathrm{xCT}$-expressing gastric cancer patients had a lower OS (hazard ratio [HR]: 1.48, 1.04-2.31, $\log$ rank $p=$ 0.027 , Figure 2E) and a lower PFS (HR: 1.43, 1.01-2.02, $\log$ rank $p=0.043$, Figure $2 \mathrm{~F}$ ) than low xCT-expressing patients. These results suggested that high $\mathrm{xCT}$ expression is a poor prognostic factor for gastric cancer patients receiving adjuvant chemotherapy treatment.

\section{Mitochondrial dysfunction enhances cisplatin resistance and increases $\mathrm{x} C \mathrm{~T}$ expression in human gastric cancer cells}

Three human gastric cancer cell lines (SC-M1, AGS, and AZ521) were treated with $0.5-5 \mu \mathrm{g} / \mathrm{ml}$ oligomycin (a Complex V ATPase inhibitor) or $1-5 \mu \mathrm{g} / \mathrm{ml}$ antimycin A (a Complex III inhibitor) to induce mitochondrial dysfunction, as in our previous studies $[6,9,21,22]$. We found that sensitivity to cisplatin was decreased in the cells with oligomycin co-treatment, compared to those without oligomycin treatment (Figure 3A). The decrease in sensitivity to cisplatin was also observed in the gastric cancer cells treated with antimycin A (Figure 3B). In addition, we found that both oligomycin- and antimycin A-induced mitochondrial dysfunction could increase the gene expression of $\mathrm{xCT}$ (Figure 3C), and its protein expression (Figures 3D and 3E). The up-regulation of $\mathrm{xCT}$ expression by oligomycin treatment occurred in a timedependent manner (Figure 3F). Based on the function of the system $\mathrm{x}_{\mathrm{c}}^{-}$transporter, we determined intracellular GSH levels and found that intracellular GSH was increased by oligomycin-induced mitochondrial dysfunction (Figure $3 \mathrm{G})$. These results suggested that mitochondrial dysfunction could enhance cisplatin resistance and increase xCT expression in human gastric cancer cells.

\section{Inhibition and knockdown of $x C T$ reduce mitochondrial dysfunction-enhanced cisplatin resistance}

To evaluate whether the increased $\mathrm{xCT}$ expression contributed to cisplatin resistance, we used two $\mathrm{xCT}$ inhibitors (SSA and erastin) to inhibit $\mathrm{xCT}$ function. We found that both SSA and erastin could significantly reduce oligomycin-induced cisplatin resistance (Figures 4A and 4B). Antimycin A-induced cisplatin resistance was also reduced by SSA treatment (Figure 4C). We further used specific siRNA to knock down $\mathrm{xCT}$ expression and found that the knockdown of $\mathrm{xCT}$ could decrease oligomycin-induced cisplatin resistance (Figures 4D and 4E). Moreover, we used BSO to inhibit the biosynthesis of GSH, and found that BSO could reduce oligomycininduced cisplatin resistance (Figure 4F). These results suggested that increased $\mathrm{xCT}$ expression contributed to mitochondrial dysfunction-enhanced cisplatin resistance.

\section{Activation of the eIF2 $\alpha$-ATF4 pathway contributes to mitochondrial dysfunction- induced $\mathrm{xCT}$ expression and high $\mathrm{xCT}$ expression in cisplatin-resistant cancer cells}

Because it was reported that the eIF2 $\alpha$-ATF4 pathway increases $\mathrm{xCT}$ expression in response to stress conditions [16], we evaluated whether this pathway was involved in mitochondrial dysfunction-induced $\mathrm{xCT}$ expression. Immunoblot results showed that both the levels of phosphorylated eIF $2 \alpha$ and ATF4 protein expression were increased by oligomycin (Figure 5A) and antimycin A (Figure 5B), respectively. These results indicated that the eIF2 $\alpha$-ATF 4 pathway was activated by mitochondrial dysfunction. We also found that the cisplatin-resistant cells had increased eIF $2 \alpha$ phosphorylation and ATF4 protein expression (Figure 5C). In addition, the knockdown of eIF2 $\alpha$ by siRNA (sieIF $2 \alpha$ ) could reduce both ATF 4 and $\mathrm{xCT}$ protein expression (Figure 5D) and could sensitize cisplatin-resistant cells to cisplatin (Figure 5E). 
A

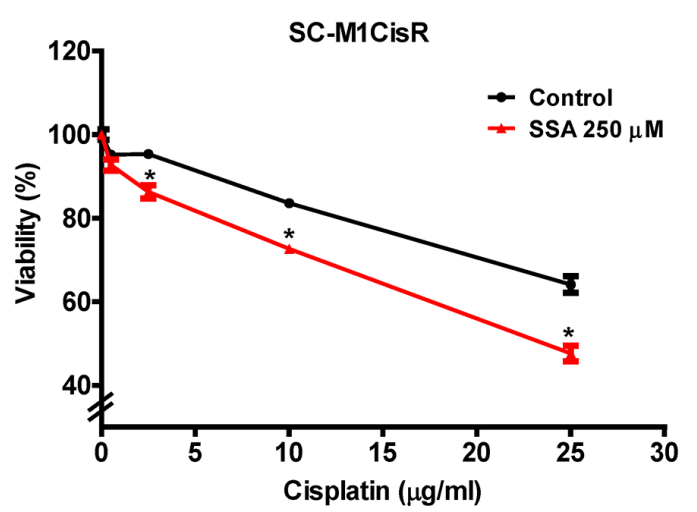

C
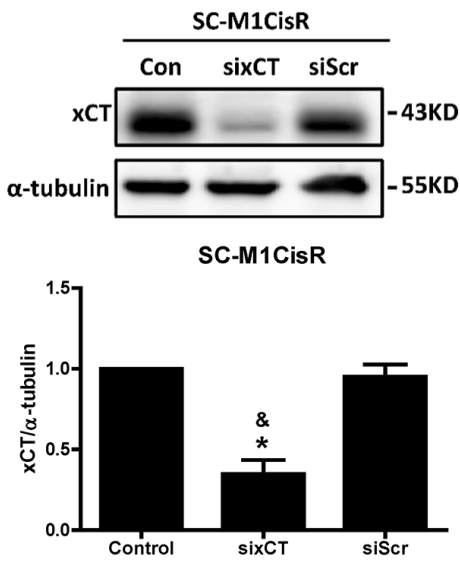

$\mathbf{E}$

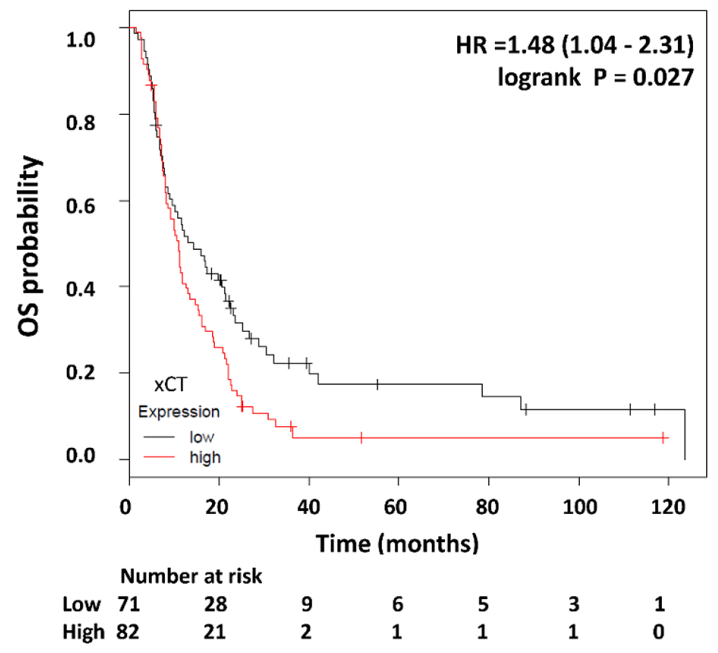

B

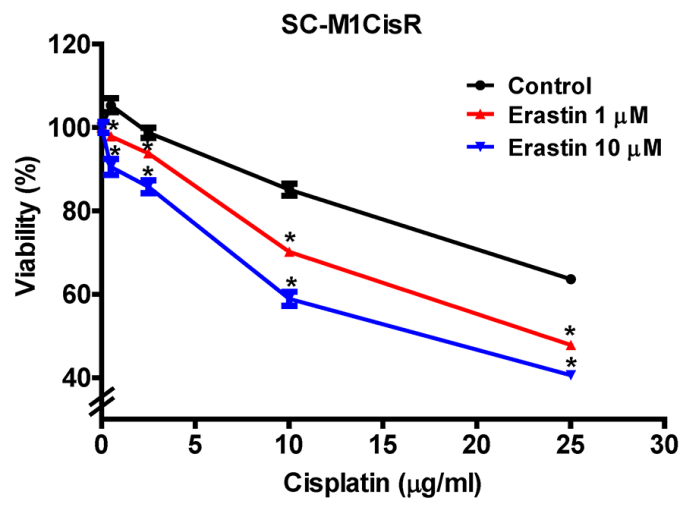

D

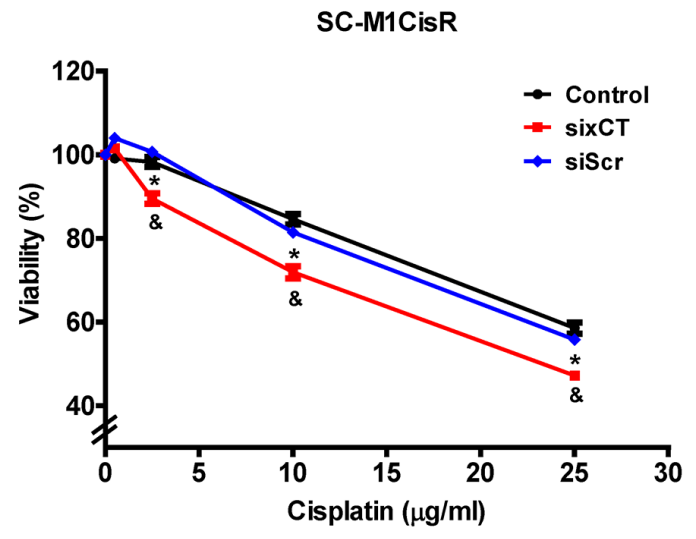

$\mathbf{F}$

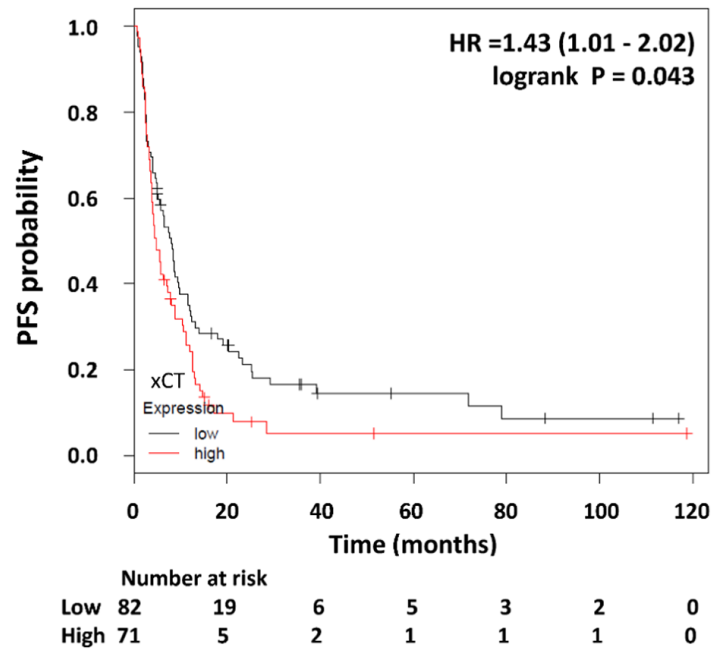

Figure 2: Inhibition and knockdown of $\mathrm{xCT}$ increase the cisplatin sensitivity of cisplatin-resistant gastric cancer cells, and high $\mathrm{xCT}$ expression is a poor prognostic factor in gastric cancer patients under adjuvant chemotherapy treatment. A. The SC-M1CisR cells were treated with cisplatin and SSA for $48 \mathrm{~h}$. The cell viability was determined by MTT assay. B. The SC-M1CisR cells were treated with cisplatin and erastin for $48 \mathrm{~h}$. The cell viability was determined by SRB assay. C. Specific siRNA against $\mathrm{xCT}\left(60\right.$ pmol for $4 \times 10^{5}$ cells in a 6-cm dish) was used to knock down $\mathrm{xCT}$ in the SC-M1CisR cells, and the protein level of xCT was analyzed by Western blot analysis. (siRNA for non-target sequence, siScramble, siScr) D. The xCT-silenced SC-M1CisR cells (sixCT) and the control SC-M1CisR cells were treated with cisplatin for $48 \mathrm{~h}$. The cell viability was determined by SRB assay. (E, F) The Kaplan-Meier survival analyses show the effects of xCT expression on overall survival (OS) E. and progression-free survival (PFS) F. in the subgroup of gastric cancer patients (5-FU-based adjuvant gastric cancer patients). Data represent the mean \pm SEM of three independent experiments. ${ }^{*} p<0.05$, compared to the control group; \& $p<0.05$, compared to the individual siScr group. 
A
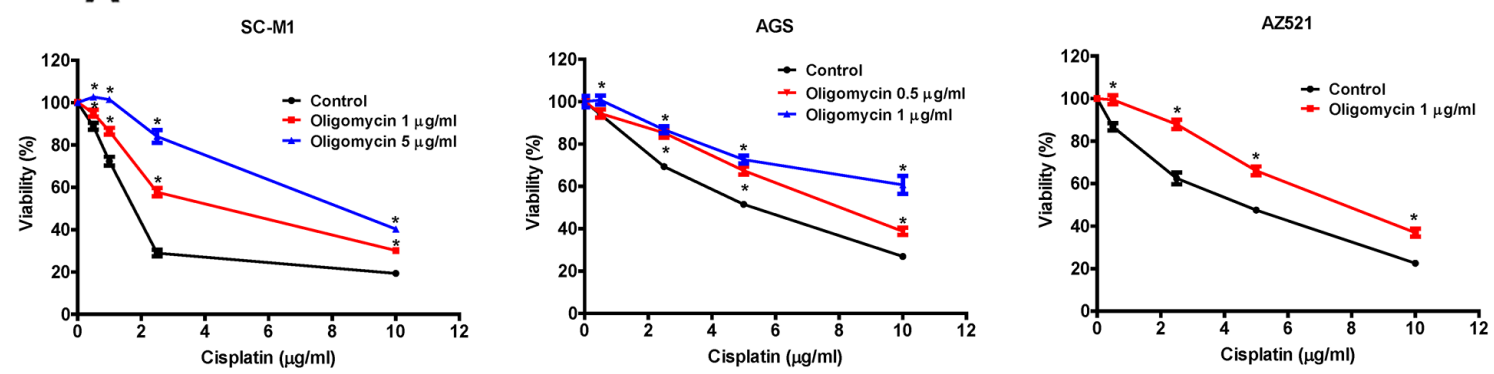

B

D

E
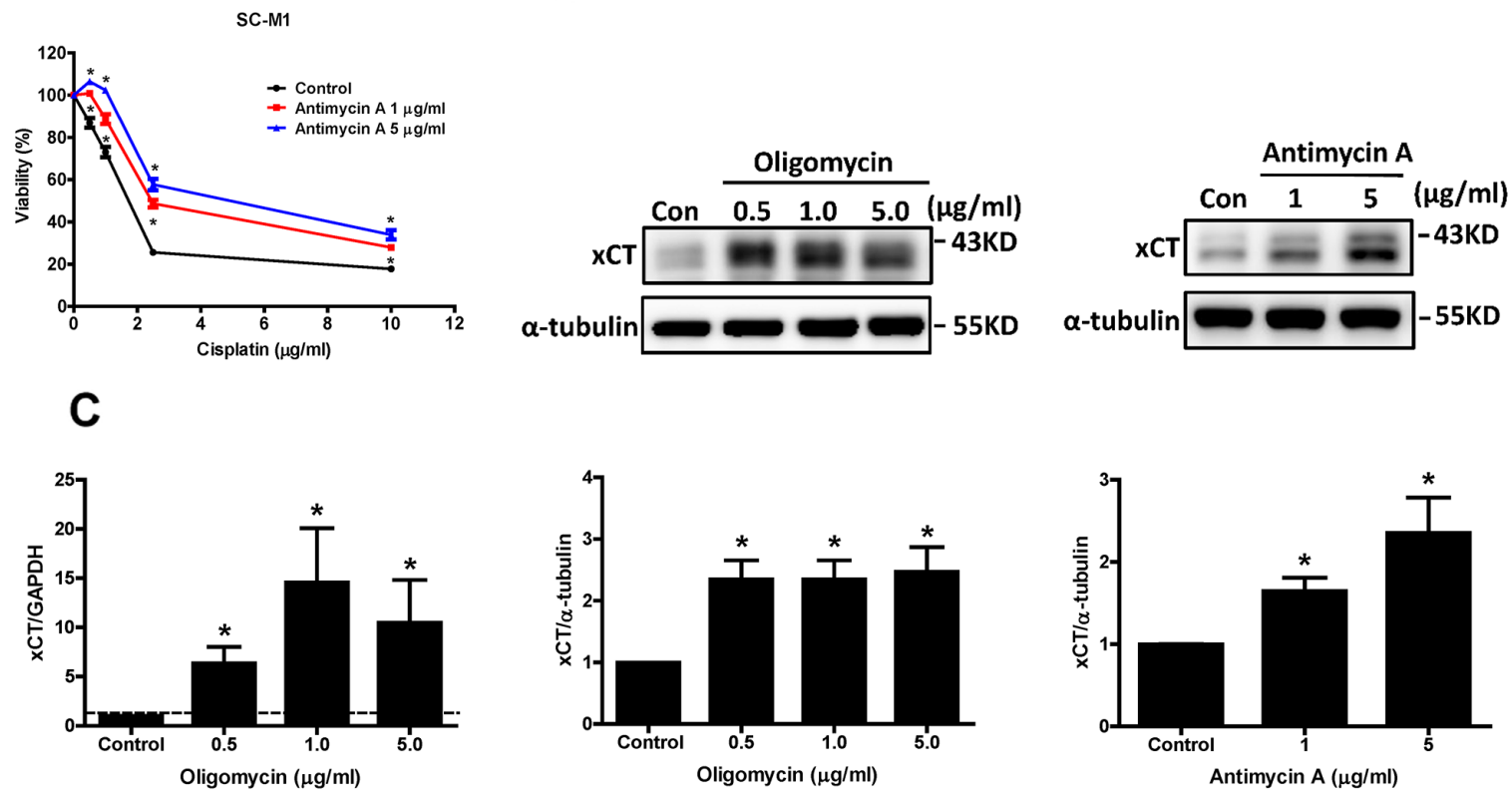

$$
\mathbf{F}
$$
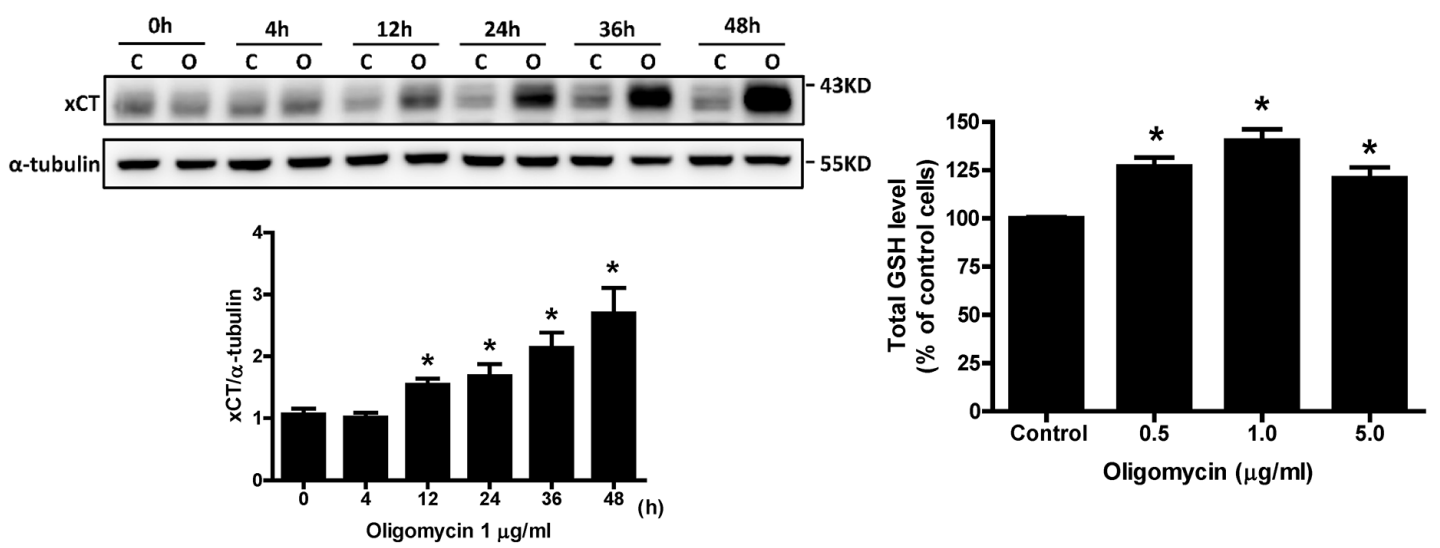

Figure 3: Mitochondrial dysfunction enhances cisplatin resistance and increases $\mathrm{xCT}$ expression in human gastric cancer cells. A. Human gastric cancer cells SC-M1, AGS, and AZ521 were treated with cisplatin and oligomycin for 48 h. B. The SC-M1 cells were treated with cisplatin and antimycin A for $48 \mathrm{~h}$. The cell viability was determined by SRB assay. C. qRT-PCR analysis of xCT mRNA in the SC-M1 cells under oligomycin treatment for $24 \mathrm{~h}$. qRT-PCR values were normalized to GAPDH mRNA. (D, E) Western blot analysis of xCT protein expression in the SC-M1 cells under oligomycin D. and antimycin A E. treatments for $24 \mathrm{~h}$, respectively. F. The SC-M1 cells were treated with oligomycin for 0-48 h (C: control; O: oligomycin). The changes of xCT protein expression were analyzed by Western blot. G. For glutathione (GSH) assay, the SC-M1 cells were seeded at a density of 1 x $10^{6}$ cells per 10-cm dish. After oligomycin treatments for $24 \mathrm{~h}$, the cells were collected, and the total cellular GSH level was analyzed with a GSH assay kit. Data represent the mean \pm SEM of three independent experiments. ${ }^{*} p<0.05$, compared to the control group. 


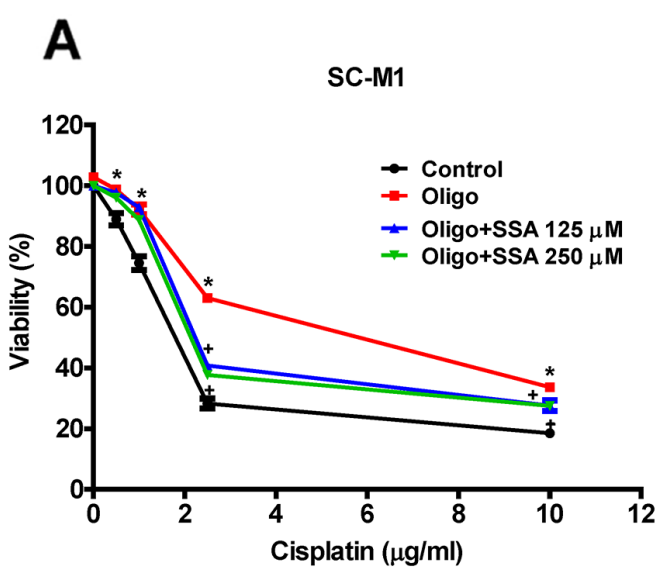

B

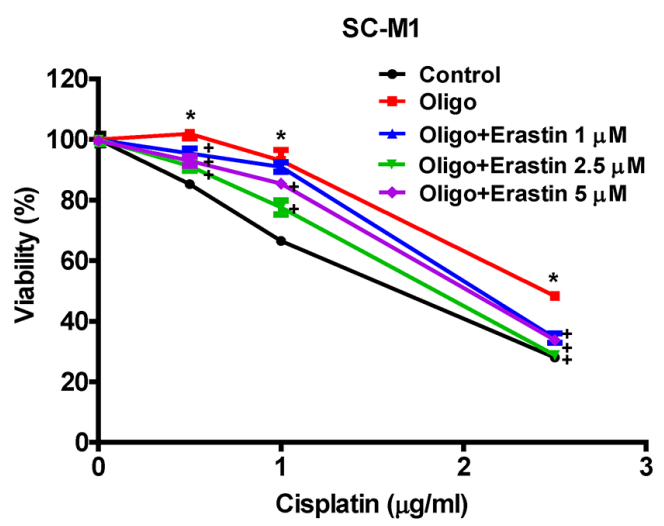

C

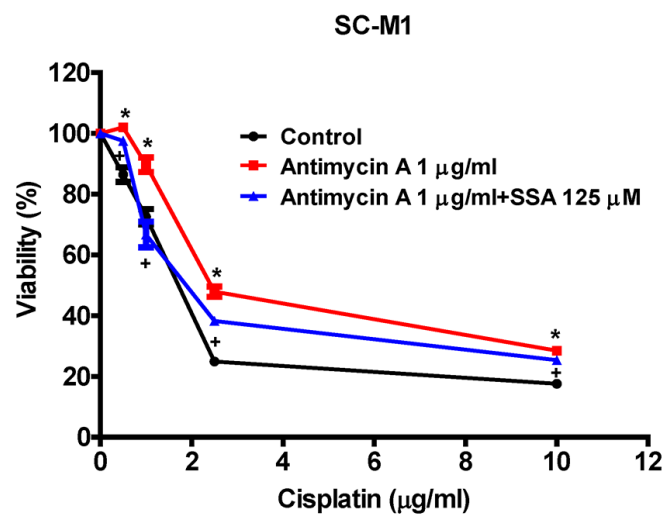

D

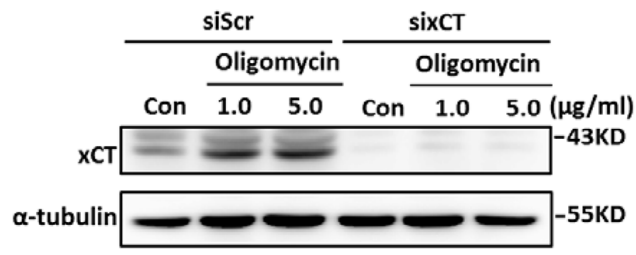

$\mathbf{E}$

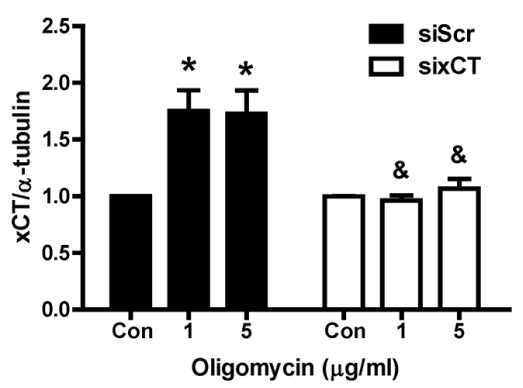

SC-M1

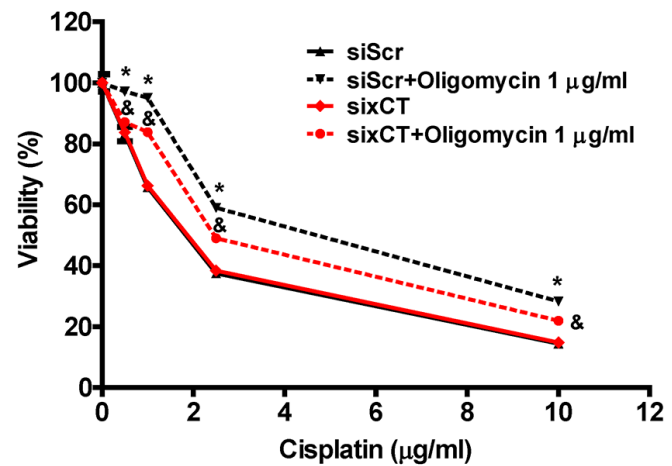

$\mathbf{F}$

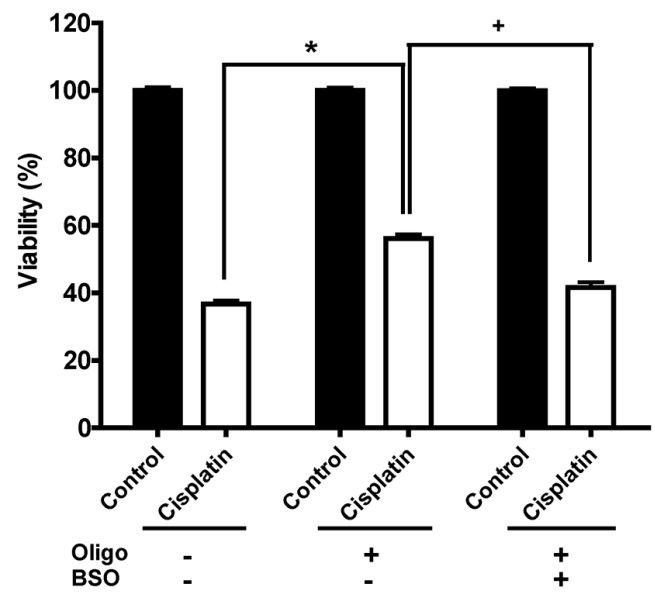

Figure 4: Inhibition and knockdown of $\mathrm{xCT}$ reduce mitochondrial dysfunction-enhanced cisplatin resistance. (A, B) The SC-M1 cells were treated with oligomycin, cisplatin, and sulfasalazine (SSA) A. or erastin B. for $48 \mathrm{~h}$. (Oligo: oligomycin $1 \mu \mathrm{g} / \mathrm{ml}$ ). C. The SC-M1 cells were treated with antimycin A, cisplatin, and SSA for $48 \mathrm{~h}$. The cell viability was determined by SRB assay. D. Western blot analysis of the $\mathrm{xCT}$ protein expression in the $\mathrm{xCT}$-silenced SC-M1 cells (sixCT) and the control cells (siRNA for non-target sequence, siScramble, siScr) under oligomycin treatment for $24 \mathrm{~h}$. E. Specific siRNA (60 pmol for $4 \times 10^{5}$ cells in a $6-\mathrm{cm}$ dish) against xCT was used to knock down xCT in the SC-M1 cells. The cells were treated with oligomycin and cisplatin for $48 \mathrm{~h}$. F. The SC-M1 cells were treated with oligomycin, cisplatin $(2.5 \mu \mathrm{g} / \mathrm{ml})$ and/or buthionine sulphoximine (BSO, $0.5 \mathrm{mM})$ for $48 \mathrm{~h}$. The cell viability was determined by SRB assay. Data represent the mean \pm SEM of three independent experiments. ${ }^{*} p<0.05$, compared to the control group; $+p<0.05$, compared to the oligomycin alone group; \& $p<0.05$, compared to the individual siScr group. 
A

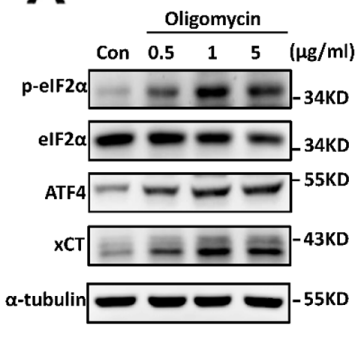

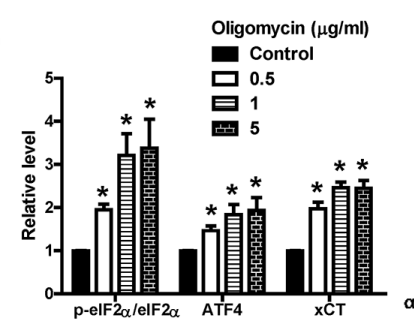

B
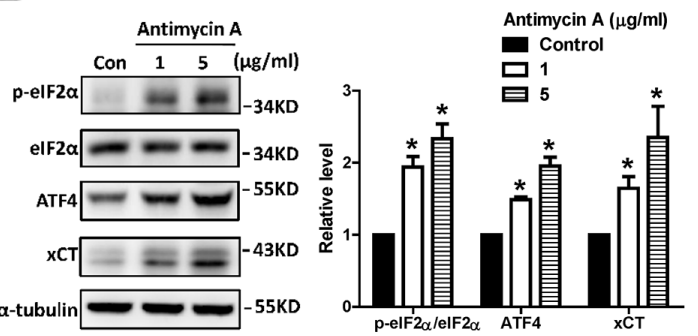

C
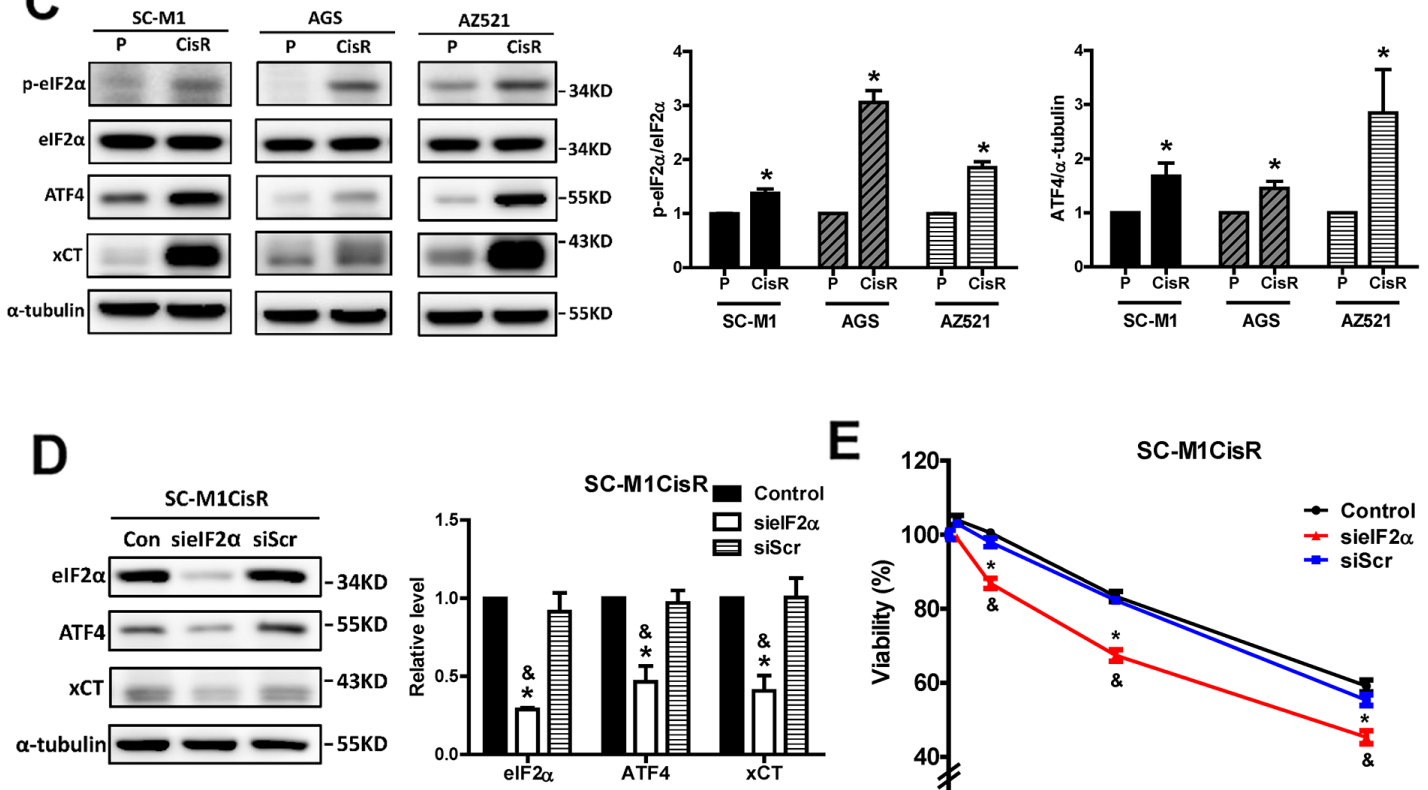

E

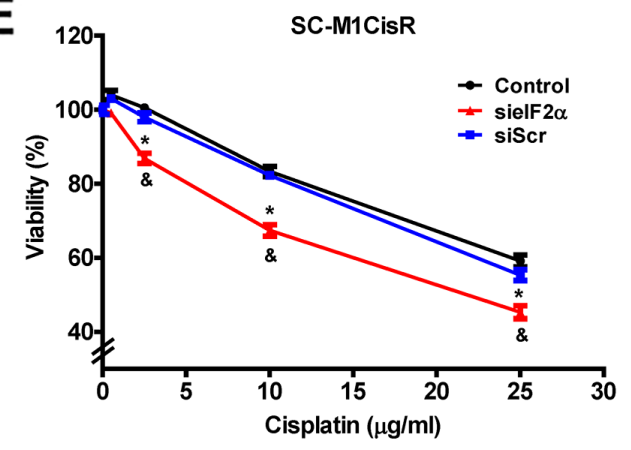

$\mathbf{F}$
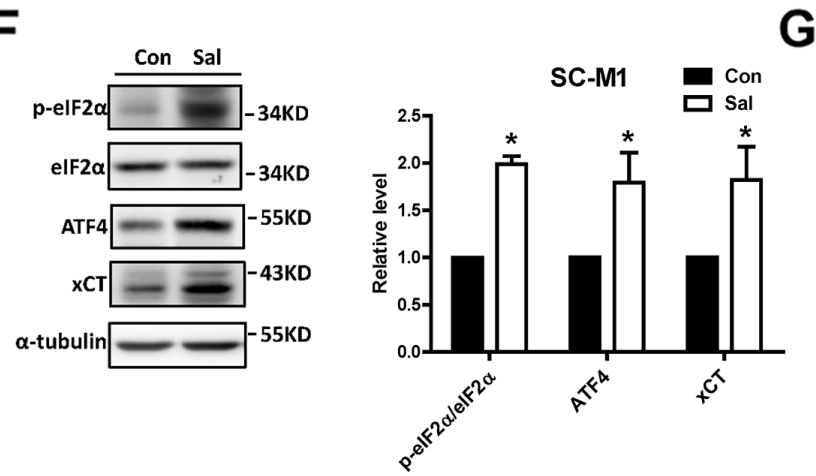

$\mathbf{G}$

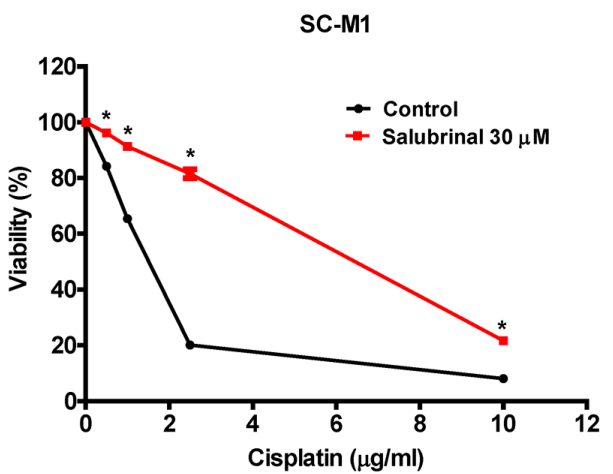

Figure 5: Activation of the eIF2 $\alpha$-ATF4 pathway contributes to mitochondrial dysfunction-induced xCT expression and high $\mathrm{xCT}$ expression in cisplatin-resistant cancer cells. A, B. Western blot analysis of the eIF2 $\alpha$-ATF4-xCT pathway in SCM1 cells under oligomycin (A) and antimycin A (B) treatments for $24 \mathrm{~h}$, respectively. C. Western blot analysis of the eIF2 $\alpha$-ATF4-xCT pathway between parental and the cisplatin-resistant gastric cancer cells. The immunoblot values were normalized to $\alpha$-tubulin. The eIF2 $\alpha$ ATF4-xCT pathway was determined by Western blot with specific antibodies against phosphorylated eIF2 $\alpha$, eIF2 $\alpha$, ATF4, and xCT. D. The specific eIF2 $\alpha$ siRNA (60 pmol for 4 × $10^{5}$ cells in a 6-cm dish) was used to knock down eIF2 $\alpha$ in the SC-M1CisR gastric cancer cells, and the eIF2 $\alpha$-ATF4-xCT pathway was analyzed by Western blot analysis. E. The eIF2 $\alpha$-silenced SC-M1CisR cells (sieIF2 $\alpha$ ) and the control $\mathrm{SC}-\mathrm{M} 1 \mathrm{CisR}$ cells were treated with cisplatin for $48 \mathrm{~h}$. The cell viability was determined by SRB assay. F. The SC-M1 cells were treated with salubrinal (Sal, $30 \mu \mathrm{M})$ for $24 \mathrm{~h}$ and, the eIF2 $\alpha$-ATF4-xCT pathway was analyzed by Western blot analysis. The immunoblot values were normalized to $\alpha$-tubulin. G. The SC-M1 cells were pre-treated with $30 \mu \mathrm{M}$ salubrinal for $24 \mathrm{~h}$, followed by co-treatment with cisplatin for $48 \mathrm{~h}$. The cell viability was determined by MTT assay. Data represent the mean \pm SEM of three independent experiments. ${ }^{*} p<0.05$, compared to the control group or parental cells; \& $p<0.05$, compared to the individual siScr group. 
To elucidate whether the activation of the eIF $2 \alpha-$ ATF4 pathway per se was sufficient for the increases in $\mathrm{xCT}$ expression and cisplatin resistance, we treated the cancer cells with salubrinal (an eIF $2 \alpha$ phosphatase inhibitor) [23] to elevate the activity of this pathway. Salubrinal treatments not only increased eIF2 $\alpha$ phosphorylation and ATF4 protein expression, but also elevated $\mathrm{xCT}$ expression (Figure 5F) and cisplatin resistance (Figure 5G).

To validate further the role of ATF4 in $\mathrm{xCT}$ expression, we used siRNA to knockdown ATF4 in the parental and cisplatin-resistant SC-M1 cells. We found that ATF4 knockdown could decrease the extent of oligomycininduced $\mathrm{xCT}$ expression (Figure 6A), and could reduce oligomycin-induced cisplatin resistance (Figure 6B). Consistently, the knockdown of ATF4 in the cisplatinresistant cells could decrease xCT expression (Figure 6C) and could partly reduce cisplatin resistance (Figure 6D). Furthermore, we constructed wild-type, AARE-mutant, and ARE-mutant $\mathrm{xCT}$ promoter luciferase reporter plasmids to demonstrate the importance of the AAREs of the $\mathrm{xCT}$ promoter to the mechanism. We first assessed whether oligomycin and salubrinal increase the expression levels of $\mathrm{xCT}$ mRNA and protein and activate the eIF2 $\alpha$-ATF4xCT pathway in HEK293T cells (Figures 6E and 6F). Importantly, we found that oligomycin and salubrinal could increase the wild-type $\mathrm{xCT}$ promoter reporter activity but could not significantly increase the AARE-mutant $\mathrm{xCT}$ promoter reporter activity (Figure 6G). Moreover, ATF4 knockdown could reduce the extent of oligomycin-induced $\mathrm{xCT}$ promoter reporter activity (Figures $6 \mathrm{H}$ and 6I). These results suggested that ATF4 was involved in the mechanism. In contrast, oligomycin and salubrinal could increase the ARE-mutant $\mathrm{xCT}$ promoter reporter activity (Figure $6 \mathrm{~J})$. In addition, the protein expression of $\mathrm{Nrf} 2$ was not significantly increased by oligomycin treatment or in the cisplatin-resistant SC-M1 cells (Supplementary Figures $\mathrm{S} 1 \mathrm{~F}$ and $\mathrm{S} 1 \mathrm{G}$ ). These results suggested that Nrf2 was not involved in $\mathrm{xCT}$ expression in our system. Therefore, these results suggested that the activation of the eIF $2 \alpha-\mathrm{ATF} 4$ pathway could increase $\mathrm{xCT}$ expression and contribute to mitochondrial dysfunction-enhanced cisplatin resistance.

\section{GCN2, but not PERK, participates in the eIF2 $\alpha$ - ATF4-xCT pathway, in cisplatin resistance in response to mitochondrial dysfunction and in cisplatin-resistant cells}

We further explored whether the eIF2 $\alpha$ kinase PERK was involved in mitochondrial dysfunction-enhanced cisplatin resistance. We found that oligomycin treatments slightly increased PERK phosphorylation (Figure 7A). However, a specific PERK inhibitor (GSK2606414) did not significantly reduce oligomycin-induced cisplatin resistance (Figure 7B). Moreover, the PERK phosphorylation levels were not significantly increased in the cisplatin- resistant cancer cells (Figure 7C). The change in PERK phosphorylation in cisplatin-resistant AZ521 cells might result from the variation of total PERK protein level. The detailed reason remains unclear. In addition, GSK2606414 treatment did not significantly sensitize the cisplatinresistant cancer cells to cisplatin (Figure 7D). We further used the genetic knockdown of PERK to confirm the role of PERK in mitochondrial dysfunction-enhanced cisplatin resistance. The results revealed that PERK knockdown did not decrease the oligomycin-induced activation of the eIF2 $\alpha$-ATF4-xCT pathway (Figure 7E) and did not reduce oligomycin-induced cisplatin resistance (Figure 7F). In the cisplatin-resistant cells, PERK knockdown could not decrease the phosphorylation of eIF $2 \alpha$ or the expression levels of ATF4 and xCT (Figure 7G), and it could not reduce cisplatin resistance (Figure $7 \mathrm{H}$ ). These results suggested that PERK might not play a major role in oligomycininduced cisplatin resistance.

In contrast, we found that oligomycin treatments could increase GCN2 phosphorylation and the phosphorylated GCN2 level was higher in the cisplatinresistant cells than in the parental cells (Figures 8A and 8B). In addition, the knockdown of GCN2 decreased the oligomycin-induced activation of the eIF2 $\alpha$-ATF4-xCT pathway (Figure 8C) and reduced oligomycin-induced cisplatin resistance (Figure 8D). These results suggested that GCN2 was involved in the mitochondrial dysfunctioninduced eIF2 $\alpha$-ATF4-xCT pathway and in cisplatin resistance. Consistently, the knockdown of GCN2 in the cisplatin-resistant cells could decrease the phosphorylation of eIF $2 \alpha$ and the expression levels of ATF4 and xCT (Figure $8 \mathrm{E}$ ) and could partly reduce cisplatin resistance (Figure 8F). These results suggested that GCN2 was involved in the activation of the eIF $2 \alpha$-ATF4-xCT pathway and cisplatin resistance in human gastric cancer cells.

\section{ROS mediates the mitochondrial dysfunction- induced activation of the GCN2-eIF2 $\alpha$-ATF4- xCT pathway}

Mitochondrial electron transport chains constitute the major intracellular ROS source and increased ROS levels are usually obtained in cells with mtDNA mutations and mitochondrial dysfunction [4]. Moreover, mitochondrial dysfunction due to oligomycin could increase intracellular (DCF) and mitochondrial (MitoSOX Red) ROS (Figure 9A). Hence, we further determined whether ROS participated in mitochondrial dysfunctioninduced GCN2 activation. We found that the antioxidant NAC could reverse the phosphorylation of GCN2 by oligomycin treatment (Figure 9B). Furthermore, we found that NAC could reverse the activation of the GCN2-ATF4eIF $2 \alpha-x C T$ pathway by oligomycin (Figure 9C). These results indicated that ROS mediated the mitochondrial dysfunction-induced activation of the GCN2-ATF4eIF $2 \alpha-x$ CT pathway. 

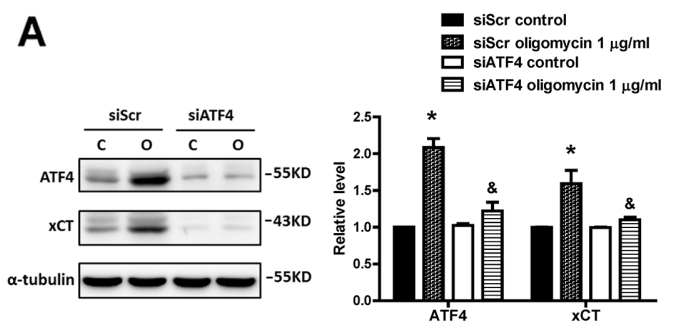

C
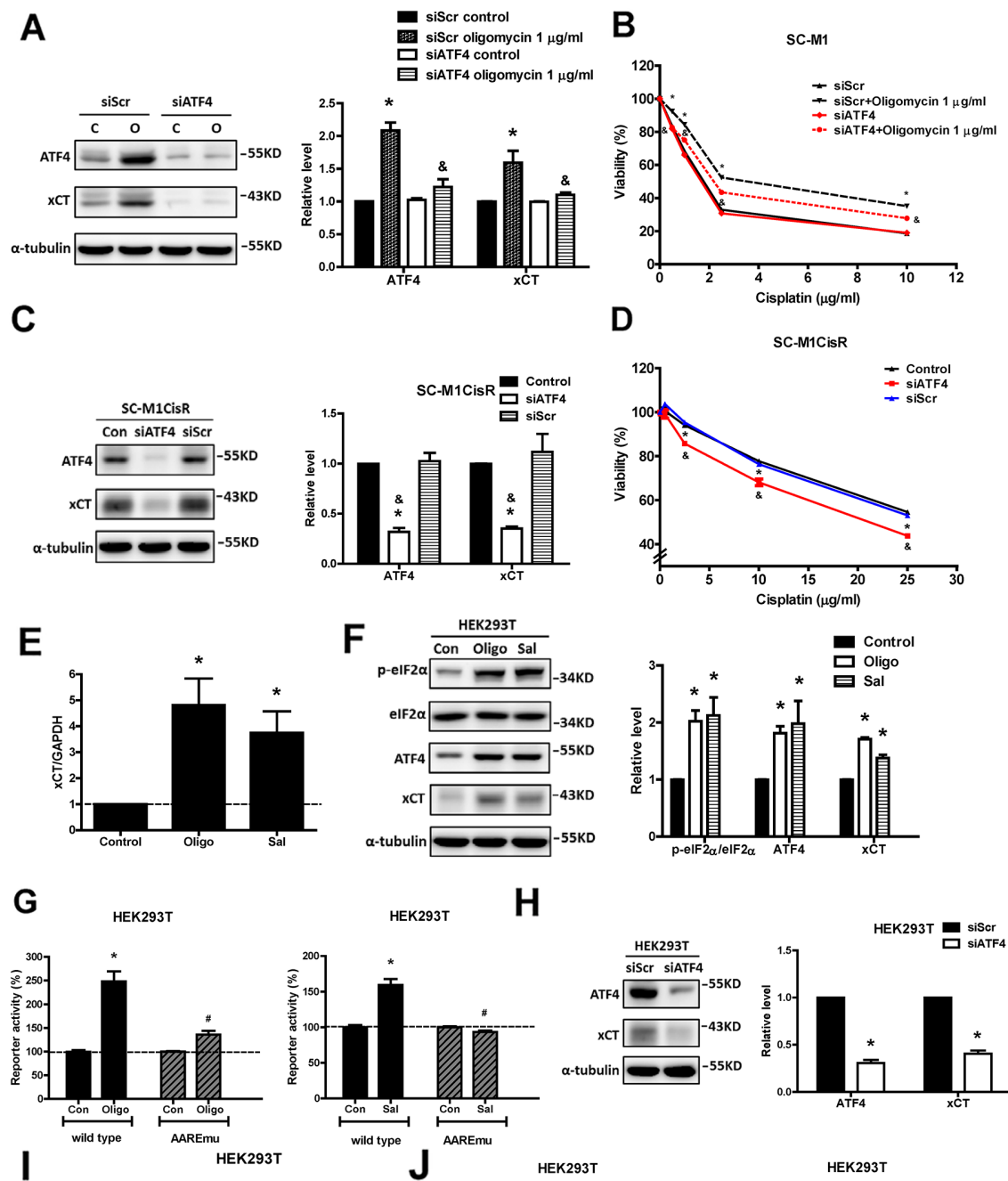

H
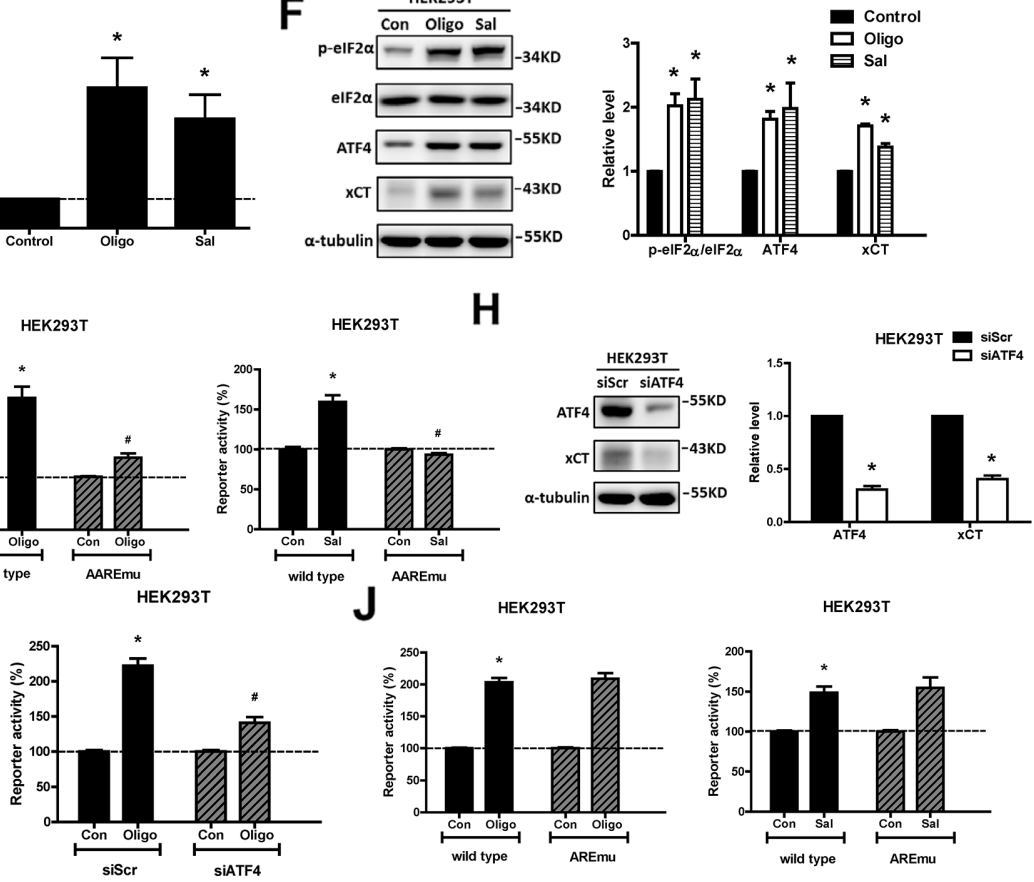

Figure 6: ATF4, an eIF2 $\alpha$ downstream factor, is responsible for mitochondrial dysfunction-induced xCT expression and cisplatin resistance. A. Western blot analysis of the expressions of ATF4 and $\mathrm{xCT}$ in the ATF4-silenced SC-M1 cells (siATF4) and the control cells (siRNA for non-target sequence, siScramble, siScr) under oligomycin treatment for $24 \mathrm{~h}$. The immunoblot values were normalized to $\alpha$-tubulin. B. The specific siRNA (100 pmol for $4 \times 10^{5}$ cells in a $6-\mathrm{cm}$ dish) against ATF4 was used to knock down ATF4 in the SC-M1 cells. The cells were treated with oligomycin and cisplatin for $48 \mathrm{~h}$. The cell viability was determined by SRB assay. C. The ATF4 siRNA (100 pmol for $4 \times 10^{5}$ cells in a 6-cm dish) was used to knock down ATF4 in the SC-M1CisR cells and the expressions of ATF4 and xCT were analyzed by Western blot analysis. D. The ATF4-silenced SC-M1CisR cells (siATF4) and the control SC-M1CisR cells were treated with cisplatin for $48 \mathrm{~h}$. The cell viability was determined by SRB assay. E. qRT-PCR analysis of xCT mRNA in the HEK293T cells under $1 \mu \mathrm{g} / \mathrm{ml}$ oligomycin and $30 \mu \mathrm{M}$ salubrinal treatments for $24 \mathrm{~h}$. qRT-PCR values were normalized to GAPDH mRNA. F. Western blot analysis of the eIF2 $\alpha$-ATF4-xCT pathway in the HEK293T cells under $1 \mu \mathrm{g} / \mathrm{ml}$ oligomycin and $30 \mu \mathrm{M}$ salubrinal treatments for $24 \mathrm{~h}$. G, J The wild-type $\mathrm{xCT}$ promoter luciferase reporter (G, J), AARE-mutant (AAREmu) $\mathrm{xCT}$ promoter luciferase reporter (G) and AREmutant (AREmu) xCT promoter luciferase reporter $(\mathrm{J})$ constructs were transfected using Turbofect transfection reagent. After transfection, cells were incubated with $1 \mu \mathrm{g} / \mathrm{ml}$ oligomycin and $30 \mu \mathrm{M}$ salubrinal for $24 \mathrm{~h}$. The reporter activity was normalized with EGFP. H. Western blot analysis of the expression levels of ATF4 and xCT in the ATF4-silenced HEK293T cells (siATF4). I. The specific siRNA (20 pmol for $4 \times 10^{5}$ cells in 6-cm dish) against ATF4 was used to knock down ATF4 in the HEK293T cells. After ATF4 knockdown, cells were seeded at a density of $2 \times 10^{5}$ cells per well in 6-well plates and further transfected the wild-type $\mathrm{xCT}$ promoter luciferase reporter construct. After transfection, cells were incubated with $1 \mu \mathrm{g} / \mathrm{ml}$ oligomycin for $24 \mathrm{~h}$. Data represent the mean \pm SEM of three independent experiments. ${ }^{*} p<0.05$, compared to the control group or parental cells; \& $p<0.05$, compared to the individual siScr group; $\# p<0.05$, compared to the oligomycin- or salubrinal-treated group. 

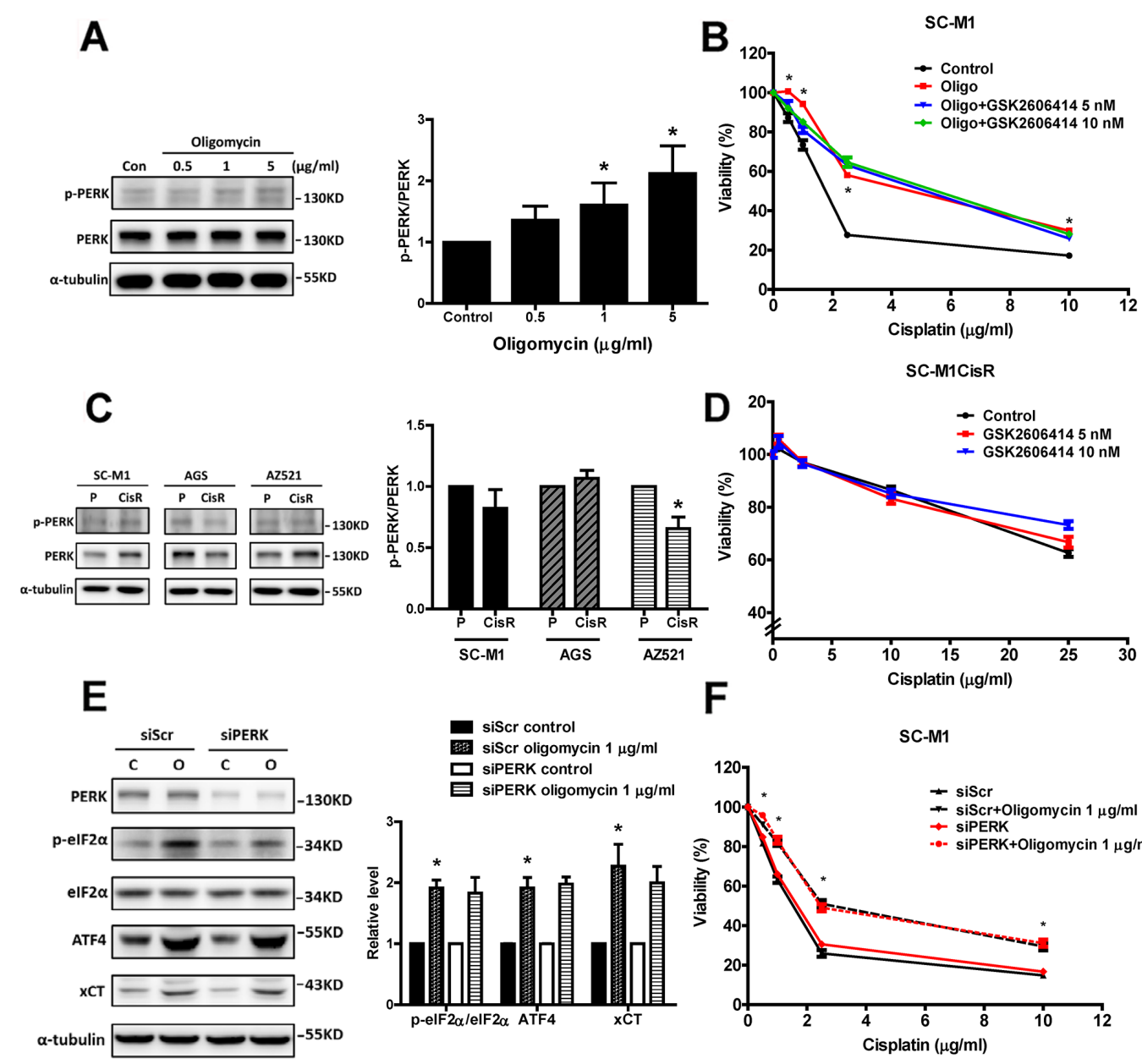

$\mathbf{F}$
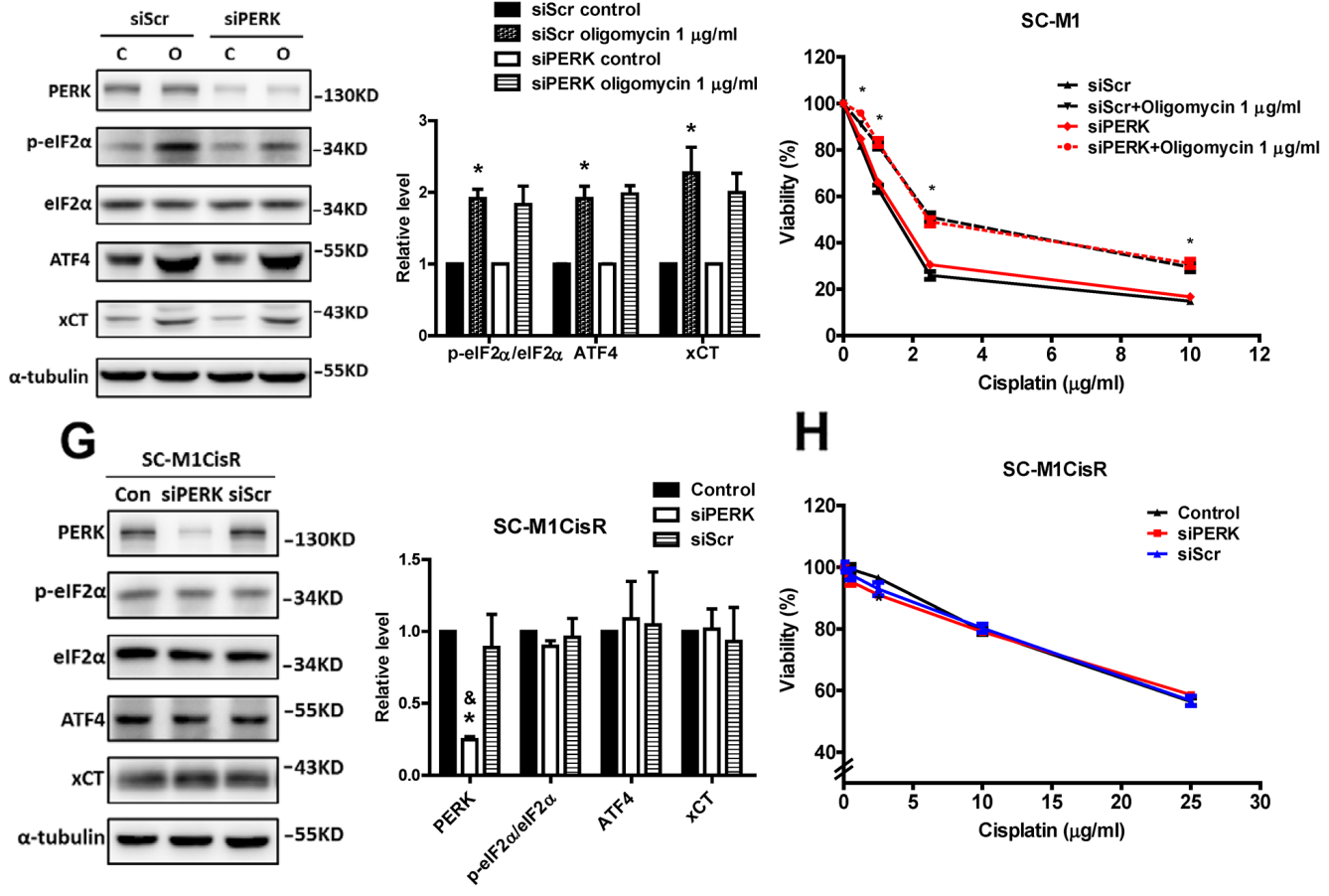

Figure 7: PERK does not participate in the eIF2 $\alpha$-ATF4-xCT pathway or cisplatin resistance in response to mitochondrial dysfunction or in cisplatin-resistant cells. A. Western blot analysis of PERK activation in the SC-M1 cells under oligomycin treatment for $24 \mathrm{~h}$. The activation of PERK was determined by Western blot with specific antibodies against phosphorylatedPERK (p-PERK) and PERK. The immunoblot values were normalized to $\alpha$-tubulin. B. The SC-M1 cells were treated with oligomycin (Oligo, oligomycin $1 \mu \mathrm{g} / \mathrm{ml}$ ), cisplatin, and GSK2606414 (PERK inhibitor) for 48h. The cell viability was determined by SRB assay. C. Western blot analysis of PERK activation between the parental and the cisplatin-resistant gastric cancer cells. The immunoblot values were normalized to $\alpha$-tubulin. D. The SC-M1CisR cells were treated with GSK2606414 and cisplatin for $48 \mathrm{~h}$. The cell viability was determined by SRB assay. E. Western blot analysis of the eIF2 $\alpha$-ATF4-xCT pathway in the PERK-silenced SC-M1 cells (siPERK) and the control cells (siRNA for non-target sequence, siScramble, siScr) under oligomycin treatments for $24 \mathrm{~h}$. The immunoblot values were normalized to $\alpha$-tubulin. F. The specific siRNA (100 pmol for 4 x $10^{5}$ cells in 6 -cm dish) against PERK was used to knock down PERK in the SC-M1 cells. The cells treated with oligomycin and cisplatin for $48 \mathrm{~h}$. The cell viability was determined by SRB assay. G. The PERK siRNA (100 pmol for $4 \times 10^{5}$ cells in a 6-cm dish) was used to knock down PERK in the SC-M1CisR cells, and the eIF2 $\alpha-A T F 4-x C T$ pathway was analyzed by Western blot analysis. H. The PERK-silenced SC-M1CisR cells (siPERK) and the control SC-M1CisR cells were treated with cisplatin for $48 \mathrm{~h}$. The cell viability was determined by SRB assay. Data represent the mean $\pm \mathrm{SEM}$ of three independent experiments. ${ }^{*} p<0.05$, compared to the control group or parental cells; $\& p<0.05$, compared to the individual siScr group. 

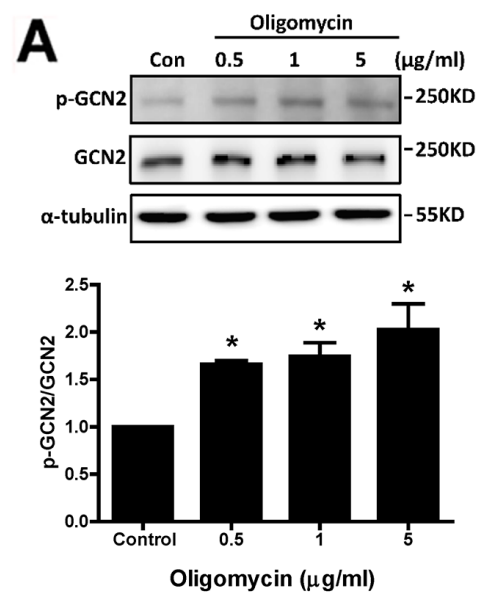

C
B
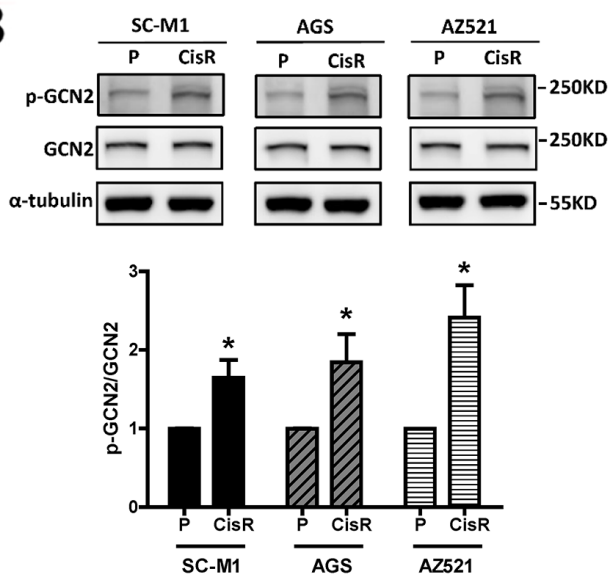

D

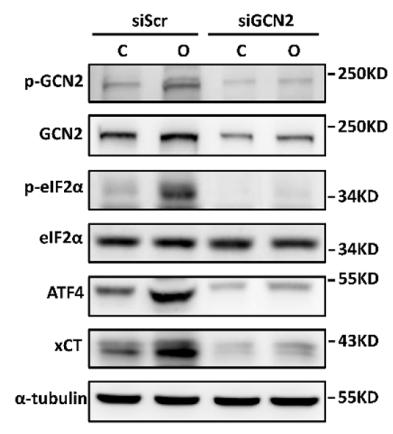

$\mathbf{E}$

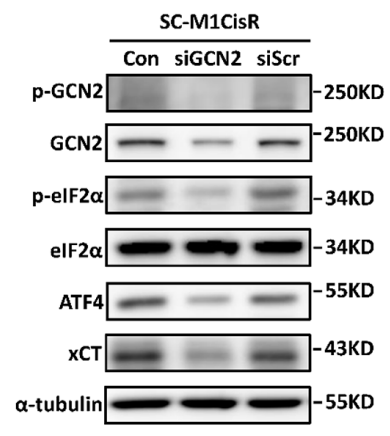

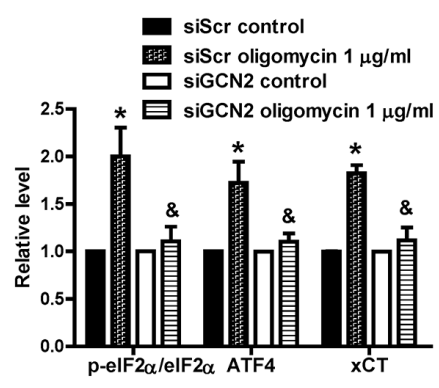

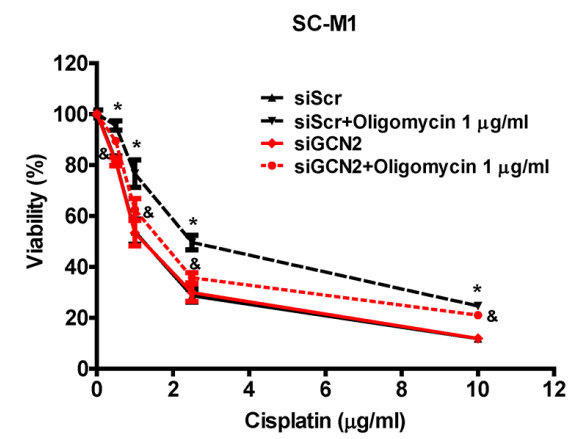

$\mathbf{F}$

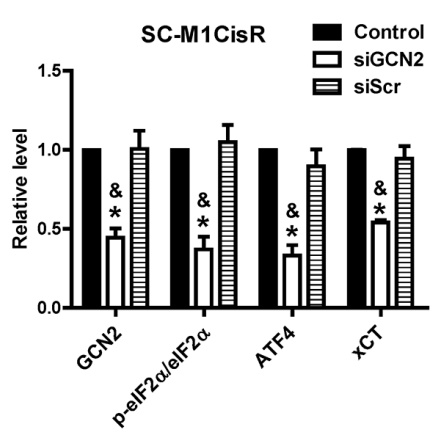

SC-M1CisR

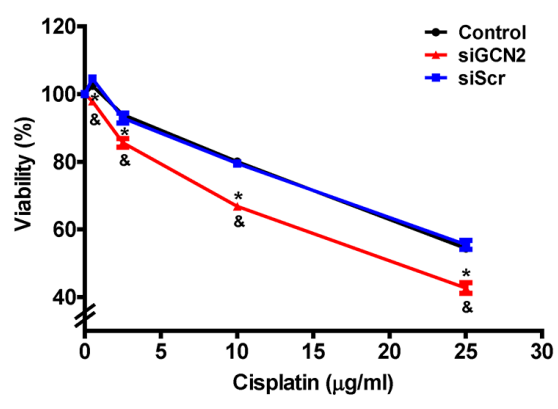

Figure 8: GCN2 participates in the eIF2 $\alpha$-ATF4-xCT pathway and cisplatin resistance in response to mitochondrial dysfunction and in cisplatin-resistant cells. A. Western blot analysis of GCN2 activation in SC-M1 cells under oligomycin treatments for $24 \mathrm{~h}$. B. Western blot analysis of GCN2 activation between the parental and the cisplatin-resistant gastric cancer cells. The activation of GCN2 was determined by Western blot with specific antibodies against phosphorylated GCN2 (p-GCN2) and GCN2. The immunoblot values were normalized to $\alpha$-tubulin. $\mathbf{C}$. Western blot analysis of the GCN2-eIF2 $\alpha$-ATF4-xCT pathway in the GCN2-silenced SC-M1 cells (siGCN2) and the control cells (siRNA for non-target sequence, siScramble, siScr) under oligomycin treatments for $24 \mathrm{~h}$. The immunoblot values were normalized to $\alpha$-tubulin. D. The specific siRNA (60 pmol for $4 \times 10^{5}$ cells in a 6-cm dish) against GCN2 was used to knock down GCN2 in the SC-M1 cells. The cells were treated with oligomycin and cisplatin for $48 \mathrm{~h}$. The cell viability was determined by SRB assay. E. The GCN2 siRNA (60 pmol for 4 x $10^{5}$ cells in a 6-cm dish) was used to knock down GCN2 in the SC-M1CisR cells, and the GCN2-eIF2 $\alpha$-ATF4-xCT pathway was analyzed by Western blot analysis. F. The GCN2-silenced SC-M1CisR cells (siGCN2) and the control SC-M1CisR cells were treated with cisplatin for $48 \mathrm{~h}$. The cell viability was determined by SRB assay. Data represent the mean \pm SEM of three independent experiments. ${ }^{*} p<0.05$, compared to the control group or parental cells; $\& p<0.05$, compared to the individual siScr group. 

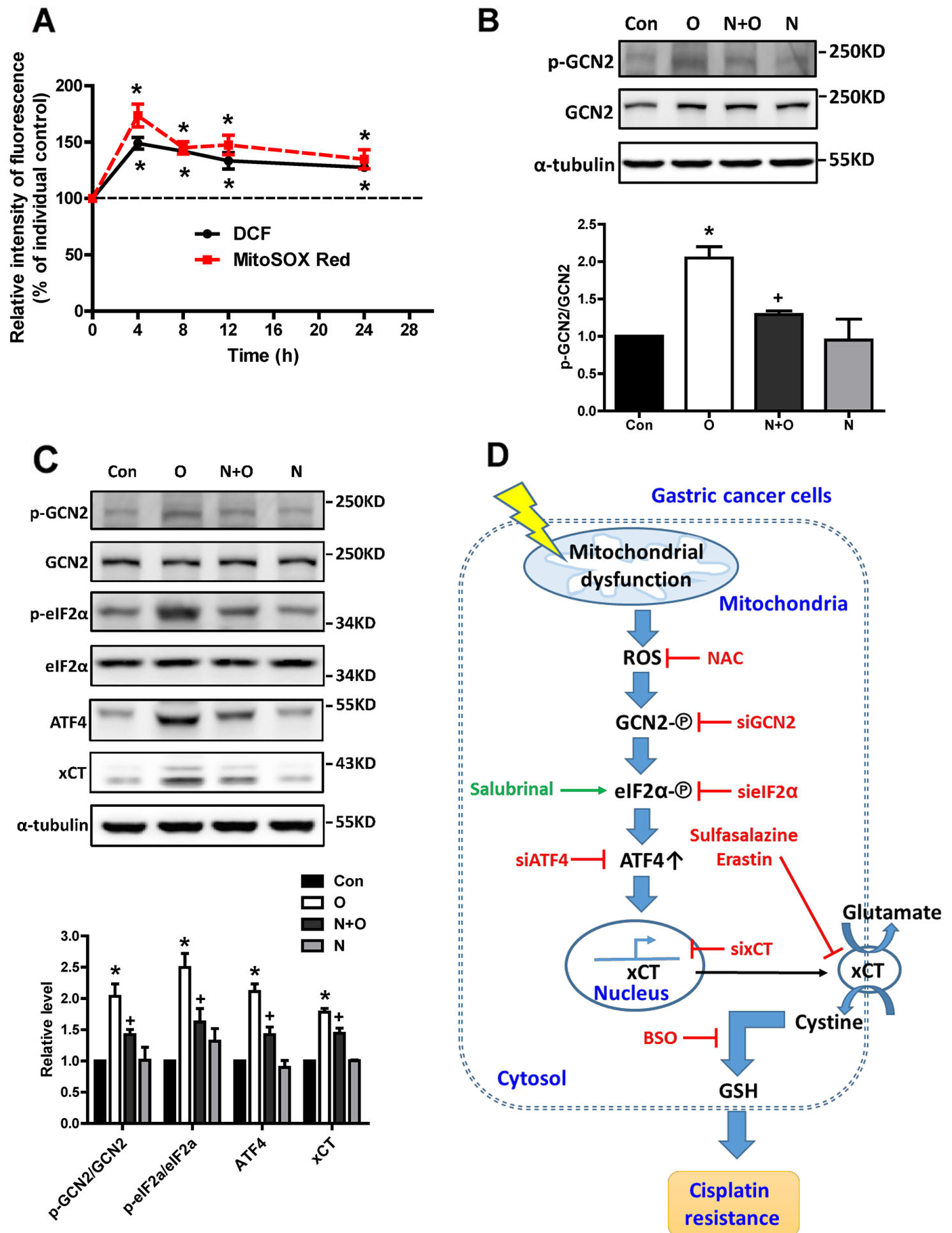

Figure 9: ROS mediates mitochondrial dysfunction-induced activation of the GCN2-eIF2 $\alpha$-ATF4-xCT pathway. A. The SC-M1 cells were treated with oligomycin for 4-24 h. After incubation for the indicated durations, the cells were stain with DCFH-dA 5 $\mu \mathrm{M}$ for $30 \mathrm{~min}$ or MitoSOX Red $10 \mu \mathrm{M}$ for $10 \mathrm{~min}$ and were further collected and analyzed by flow cytometry. B. Western blot analysis of GCN2 activation in the SC-M1 cells under oligomycin $(\mathrm{O}, 1 \mu \mathrm{g} / \mathrm{ml})$ and $\mathrm{N}$-acetyl cysteine (NAC, N, $5 \mathrm{mM})$ treatments for $4 \mathrm{~h}$. C. Western blot analysis of the GCN2-eIF2 $\alpha$-ATF4-xCT pathway in the SC-M1 cells under oligomycin $(\mathrm{O}, 1 \mu \mathrm{g} / \mathrm{ml})$ and N-acetyl cysteine (NAC, $\mathrm{N}, 5 \mathrm{mM}$ ) treatments for $24 \mathrm{~h}$. The immunoblot values were normalized to $\alpha$-tubulin. D. The scheme of the ROS-activated GCN2-eIF2 $\alpha$ ATF4-xCT pathway was involved in mitochondrial dysfunction-induced mitochondrial retrograde signaling and further conferred cisplatin resistance in human gastric cancer cells. Data represent the mean \pm SEM of three independent experiments. ${ }^{*} p<0.05$, compared to the control group; $+p<0.05$, compared to the oligomycin group. 


\section{DISCUSSION}

In this study, we demonstrated that mitochondrial dysfunction induced by oligomycin and antimycin A might enhance cisplatin resistance in human gastric cancer cells through increased xCT expression and intracellular GSH levels, as well as through the ROS activation of the GCN2-eIF2 $\alpha$-ATF4 pathway. Moreover, high xCT expression and the activation of the GCN2-eIF2 $\alpha$-ATF4 pathway were observed in cisplatin-resistant gastric cancer cells. In addition, high xCT expression in gastric cancer was associated with the poor prognosis of patients under adjuvant chemotherapy treatment. These results provided evidence for the first time to suggest that the ROS-activated GCN2-eIF2 $\alpha$-ATF4-xCT pathway is retrograde signaling that contributes to mitochondrial dysfunction-enhanced cisplatin resistance in human gastric cancer cells (Figure 9D). In our previous studies, we found that mitochondrial DNA mutations and defects in mitochondrial enzymes in gastric cancers might contribute to cancer progression and chemoresistance $[5,6]$. In this study, we further demonstrated that $\mathrm{xCT}$ might be an important link between mitochondrial dysfunction and cisplatin resistance.

The outcome of the integrated stress response induced by different mitochondrial stressors can be protective or deleterious, likely depending on the intensity and type of the mitochondrial insult or the cell type [7]. Irreversible and severe cellular damage might induce cell death. In the present study, we demonstrated that mitochondrial dysfunction induced by low concentrations of oligomycin and antimycin A could increase xCT expression by activating the eIF $2 \alpha$-ATF 4 pathway, resulting in cisplatin resistance in three different gastric cancer cell lines. Our results suggested that mild mitochondrial dysfunction could activate the protective response in human gastric cancer cells. The findings were consistent with the previous report that heteroplasmic, but not homoplasmic, mtDNA mutation could promote tumor cell growth [24]. The severe mitochondrial defects caused by homoplasmic mtDNA mutations might be deleterious to cancer development.

It has been demonstrated that the eIF $2 \alpha-A T F 4$ pathway is essential for tumor cell survival and proliferation in response to various stress conditions $[18,25]$. In addition, the increased expression of ATF4 might promote the expression of the other stress-response genes, such as CEBP homologous (CHOP), growth arrest and DNA damage-inducible protein 34 (GADD34), immunoglobulin heavy chain-binding protein (BiP), and other transcription factors [26, 27]. Therefore, it is possible that these stress-response proteins could provide the mechanisms by which cancer cells exhibit resistance to a board range of chemotherapies.

PERK and GCN2 were reported to be activated by different mitochondrial stressors $[19,28]$. In the present study, we found that GCN2, but not PERK, majorly involves in the mitochondrial dysfunction-activated eIF2 $\alpha$ ATF4-xCT pathway and cisplatin resistance in gastric cancer cells. GCN2 is a high molecular weight protein and can be activated by uncharged tRNA or impaired expression of mtDNA in different mammalian cell types $[29,30]$. Moreover, hydrogen peroxide can increase GCN2 activity through a mechanism for the tRNA synthetase domain in the C. elegans and yeasts [31-33]. Under stress conditions, GCN2 could be auto-phosphorylated and could exhibit its kinase activity [34], which phosphorylated eIF2 $\alpha$ [18]. In the present study, we demonstrated that mitochondrial dysfunction-induced ROS could activate GCN2 in human gastric cancer cells.

The system $\mathrm{x}_{\mathrm{c}}^{-}$cystine/glutamate antiporter is responsible for the cystine uptake from extracellular environment to maintain thiol-containing molecule homeostasis, particularly of GSH [13]. The imported cystine is reduced to cysteine, which is essential for GSH biosynthesis. In the present study, two inhibitors of the system $\mathrm{x}_{\mathrm{c}}{ }^{-}$antiporter (sulfasalazine and erastin) and an inhibitor of GSH biosynthesis (BSO) can reduce mitochondrial dysfunction-enhanced cisplatin resistance, suggesting that elevated intracellular GSH levels due to the system $\mathrm{x}_{\mathrm{c}}$ antiporter might contribute to chemoresistance. The GSHGSSG system not only provided an important redox buffer to scavenge cisplatin-induced oxidative stress [35], but it also deactivated cisplatin [11]. Moreover, the increased GSH might increase DNA repair and protect cancer cells from cisplatin-induced cytotoxicity [12]. Therefore, our findings provided a link between mitochondrial dysfunction and cancer progression by enhancing chemoresistance.

Increased $\mathrm{xCT}$ expression has been found to be essential for cancer proliferation and malignant progression in certain cancer cells [36, 37]. Inhibiting $\mathrm{xCT}$ attenuated stem-like cell behavior and metastatic progression in breast cancer [38]. Immunotargeting xCT increased the chemosensitivity of radioresistant cancer stem cells to doxorubicin in vivo. The expression of $\mathrm{xCT}$ at the plasma membrane of cancer cells was found to be stabilized by CD44v isoform, resulting in increased GSH synthesis [39]. Moreover, CD44v was implicated in metaplasia-carcinoma sequence progression in the stomach and conferred resistance to various types of cellular stress [40]. CD44 genetic knockdown or xCT inhibitors could suppress the development of metaplasia and subsequent gastric tumor growth. During gastric cancer development, chronic gastric inflammation and the histopathologic progression of stomach epithelium cells might constitute critical risk factors for the development of metaplasia and eventually gastric cancer. Therefore, the expression of $\mathrm{xCT}$ and CD44v might serve as biomarkers for gastric cancer progression.

In the evolution of systemic chemotherapy for gastric cancer, cisplatin-containing combinations were eventually shown to be superior to non-cisplatin- 
containing regimens, and they have become the reference regimens for advanced gastric cancer [41]. Combination chemotherapy has better response rates, but the time to progression is still only approximately 3-6 months [41]. In general, clinical trials assessing the efficacy of a variety of $2^{\text {nd }}$-line chemotherapy regimens after the failure of the $1^{\text {st }}$-line regimens have shown that the response rates are lower than those of patients without previous treatment, and the toxicity rates tend to be higher [42, 43]. Hence, chemotherapy resistance remains an obstacle to subsequent treatment despite chemotherapy for gastric cancer having acceptable clinical efficacy. xCT not only has been proposed as a possible target for cisplatin resistance but also for comprehensive chemoresistance or targeted therapy resistance [39, 44, 45]. Furthermore, another remaining barrier for chemotherapy is the selectivity for killing cancer cells based on cancer-specific features [46]. Cancer stem cells might enhance ROS scavenger capacity through increased $\mathrm{xCT}$ expression and GSH synthesis. Inhibiting $\mathrm{xCT}$ can attenuate the stemness properties and metastatic progression in various cancers $[38,39]$. Therefore, these findings suggested that $\mathrm{xCT}$ might be a poor prognostic factor for gastric cancer patients undergoing adjuvant chemotherapy treatment, and $\mathrm{xCT}$ might be a potential target for chemoresistance.

In conclusion, we identified for the first time that the ROS-activated GCN2-eIF2 $\alpha$-ATF4-xCT pathway contributes to mitochondrial dysfunction-enhanced cisplatin resistance in human gastric cancer cells. The GCN2-eIF2 $\alpha$-ATF4-xCT pathway might be a potential drug target for reducing chemoresistance and improving gastric cancer therapy.

\section{MATERIALS AND METHODS}

\section{Chemicals and reagents}

Oligomycin, sulfasalazine (SSA, an xCT inhibitor [36]), erastin (an xCT inhibitor [47]), N-acetyl cysteine (NAC), L-amino acid kit, 5-flurouracil (5-FU), antimycin A, sulforhodamine B (SRB), buthionine sulphoximine (BSO), 3-(4,5-cimethylthiazol-2-yl)-2,5-diphenyl tetrazolium bromide (MTT), acetic acid, trichloroacetic acid (TCA), aprotinin, phenylmethanesulfonyl fluoride (PMSF), sodium orthovanadate $\left(\mathrm{Na}_{3} \mathrm{VO}_{4}\right)$, dimethyl sulfoxide (DMSO), Triton X-100, Bradford reagent, bovine serum albumin (BSA), and $\alpha$-tubulin antibody were purchased from SigmaAldrich (St. Louis, MO, USA). Sodium dodecyl sulfate (SDS), Tris- $\mathrm{HCl}$ buffer, and sodium chloride $(\mathrm{NaCl})$ were purchased from Merck Millipore (Billerica, MA, USA). Cisplatin was obtained from Fresenius Kabi Oncology (Distt. Solan, H.P., India). GSK2606414 (a PERK inhibitor [48]) was purchased from Calbiochem ${ }^{\mathrm{TM}}$, Merck Millipore (Billerica, MA, USA). ON-TARGET plus ${ }^{\text {TM }}$ SMARTpool EIF2S1 (eIF2 $\alpha$ ), ATF4, SLC7A11 (xCT), EIF2AK3 (PERK), EIF2AK4 (GCN2) and non-target (scramble)
siRNAs were purchased from GE Healthcare Dharmacon (Lafayette, CO, USA). 2'7'-dichlorodihydrofluorescein diacetate (DCFH-dA) and MitoSOX Red were purchased from Molecular Probe ${ }^{\mathrm{TM}}$, Invitrogen ${ }^{\mathrm{TM}}$, Thermo Fisher Scientific (Eugene, Oregon, USA). Specific primers for real-time polymerase chain reaction (PCR) were purchased from Mission Biotech (Taipei, Taiwan). Salubrinal (an eIF2 $\alpha$ phosphatase inhibitor [23]) and the p-PERK Thr981 antibody were purchased from Santa Cruz Biotechnology (Santa Cruz, CA, USA). Antibodies against GCN2 and PERK were purchased from Cell Signaling Technology (Beverly, MA, USA). Antibodies against p-GCN2 (Thr899) and $\mathrm{xCT}$ were purchased from Abcam (Cambridge, MA, USA). ATF4 antibody was purchased from Proteintech Group (Rosemont, IL, USA). Antibodies against p-eIF2 $\alpha$ (Ser52) and eIF2 $\alpha$ were purchased from Invitrogen ${ }^{\mathrm{TM}}$, Thermo Fisher Scientific (Camarillo, CA, USA).

\section{Cell culture and establishment of resistant gastric cancer cell lines}

The human gastric cancer cell lines SC-M1, AGS, and AZ521 were cultured in RPMI 1640 medium with $10 \%$ fetal bovine serum (FBS), and $1 \%$ penicillin/ streptomycin $(\mathrm{P} / \mathrm{S})$ and were incubated in a humidified $37{ }^{\circ} \mathrm{C}$ incubator with $5 \% \mathrm{CO}_{2}$. Human embryonic kidney 293 T (HEK293T) cells were cultured in Dulbecco's modified Eagle's medium (DMEM) supplemented with $10 \%$ FBS, 2 mmol/1 L-glutamine, 10 mmol/l nonessential amino acids (NEAAs), and $1 \%$ penicillin/ streptomycin $(\mathrm{P} / \mathrm{S})$ at $37{ }^{\circ} \mathrm{C}$ in a humidified $5 \% \mathrm{CO}_{2}$ incubator. RPMI1640 and DMEM were purchased from Gibco ${ }^{\mathrm{TM}}$, Thermo Fisher Scientific (Grand Island, NY, USA). FBS, P/S, L-glutamine, and NEAA were purchased from Biological Industries (Kibbutz Beit Haemek, Israel). Cystine depletion experiments were performed by manually compounding individual cultured medium. Cystine depletion medium was formulated with or w/o amino acid RPMI 1640 (United States Biological, Swampscott, MA, USA) and the subsequent addition of the same amount of amino acid (except for cystine), according to the formula for original RPMI1640. To establish cisplatin-resistant (CisR) gastric cancer cells, the parental gastric cancer cells were treated with slowly increasing concentrations of cisplatin until the cells could tolerate the IC50 of cisplatin for 6 months. After attaining the IC50 stage, the cisplatin-resistant gastric cancer cells were cultured in medium containing the IC20 concentration of cisplatin medium for subsequent experiments (SC-M1CiSR: $0.5 \mu \mathrm{g} / \mathrm{ml}$; AGSCisR: $1 \mu \mathrm{g}$ / $\mathrm{ml}$; AZ521CisR: $1 \mu \mathrm{g} / \mathrm{ml})$. Before the function assay and drug sensitivity testing, cells were cultured in cisplatinfree medium for 3 days. The cisplatin resistance of drugresistant gastric cancer cells was routinely examined for the determination of the sensitivity to cisplatin by SRB assay every 2 months. 


\section{Determination of cell viability}

Cell viability was determined by SRB or MTT assay. Cells were seeded in 96-well cell culture plates (Corning Inc., Corning, NY, USA) at a density of 30005000 cells per well and were cultured 24 hours prior to drug treatment. In the SRB assay, cells were fixed with $10 \%$ TCA after the indicated period of treatment. After washing with distilled water, cells were stained with $0.057 \%$ SRB and were washed with $1 \%$ acetic acid. The cell viability was assessed by OD determination at $510 \mathrm{~nm}$ using a microplate reader. In the MTT assay, after the indicated period, the medium was discarded and replaced with an equal volume $(200 \mu \mathrm{l})$ of fresh medium containing MTT and was incubated at $37{ }^{\circ} \mathrm{C}$ for $1 \mathrm{~h}$ in the dark. Next, the MTT medium was discarded, and DMSO was added to dissolve the formazan that was produced. The cell viability was determined by a colorimetric method using a microplate reader at the absorption wavelength of $570 \mathrm{~nm}$.

\section{Determination of intracellular glutathione (GSH)}

Cells were seeded in a 10 -cm dish (Corning Inc., Corning, NY, USA) at a density of $1 \times 10^{6}$ cells and were cultured $24 \mathrm{~h}$ prior to drug treatment. The sample was deproteinized with $5 \%$ 5-sulfosalicylic acid solution. The cellular level of glutathione was determined using a Glutathione Assay Kit (Sigma-Aldrich, St. Louis, MO, USA), according to the manufacturer's protocol.

\section{Real-time reverse transcription (RT)-polymerase chain reaction (q-RT PCR)}

Cellular total RNA was extracted from cells using TRIzol reagent, following the manufacturer's instructions (Invitrogen ${ }^{\mathrm{TM}}$, Thermo Fisher Scientific, Carlsbad, CA, USA). Total RNA (20 $\mu$ g) was used in the RT reaction by RevertAid ${ }^{\mathrm{TM}}$ reverse transcriptase (Thermo Fischer Scientific, Waltham, MA, USA). The real-time PCR amplifications were performed in the StepOne $^{\mathrm{TM}}$ System (Applied Biosystems ${ }^{\mathrm{TM}}$ real-time PCR Instrument, Thermo Fisher Scientific) using KAPA SYBR FAST qPCR Kits (Kapa Biosystems, Wilmington, MA, USA). The primer sequences were SLC7A11 $(\mathrm{xCT})$, forward: TCATTGGAGCAGGAATCTTCA; reverse: TTCAGCATAAGAGAAAGCTCCA; and glyceraldehyde 3-phosphate dehydrogenase (GAPDH), forward: CCGTCTAGAAAAACCTGCC; reverse: GCCAAATTCGTTGTCATACC. The reaction mixture was first denatured at $95^{\circ} \mathrm{C}$ for $3 \mathrm{~min}$. The PCR condition was $95{ }^{\circ} \mathrm{C}$ for $3 \mathrm{~s}$, and $60{ }^{\circ} \mathrm{C}$ for $30 \mathrm{~s}$ each cycle $(40$ cycles). Gene expression levels were calculated by the $2^{-\triangle \Delta \mathrm{Ct}}$ method and were normalized to the level of GADPH in each sample.

\section{Western blot analysis}

Cell lysate was extracted using radioimmunoprecipitation assay buffer (RIPA buffer: 50 $\mathrm{mM}$ Tris- $\mathrm{HCl}$ buffer, $\mathrm{pH} 7.5$, containing $0.15 \mathrm{M} \mathrm{NaCl}, 0.5$ $\%$ sodium deoxycholate, $0.5 \%$ SDS, $0.1 \%$ Triton X-100, $10 \mu \mathrm{g} / \mathrm{ml}$ aprotinin, $2 \mathrm{mM}$ ethylenediaminetetraacetic acid [EDTA], $2 \mathrm{mM} \mathrm{Na} \mathrm{VO}_{4}$, and $1 \mathrm{mM}$ PMSF). The concentration of protein was determined using Bradford reagent with BSA as the standard. Twenty micrograms of lysate protein were resolved by $8-12 \%$ SDS-PAGE, transferred onto a polyvinylidene difluoride membrane (PVDF membrane, Biotrace ${ }^{\mathrm{TM}}$, PALL Life sciences, Ann Arbor, MI, USA), and immunoblotted with antibodies. The protein contents were visualized using a chemiluminescence kit (Immobilon Western Chemiluminescence HRP Substrates, Merck-Millipore, Billerica, MA, USA). The images of Western blot were observed using a Luminescence/Fluorescence Imaging System (GE Healthcare), and the signal intensities were quantified using Multi Gauge image analysis software (Fujifilm).

\section{Small interfering (siRNA)-mediated specific gene knockdown}

Cells were seeded in 6-cm dish (Corning Inc., Corning, NY, USA) at a density of $4 \times 10^{5}$ cells and were cultured overnight in antibiotic-free complete medium. Lipofectamine RNAi MAX reagent (Invitrogen $^{\mathrm{TM}}$, Thermo Fisher Scientific, Carlsbad, CA, USA) and siRNA $(60 \mathrm{pmol})$ were diluted in OPTI-MEM medium $\left(\mathrm{Gibco}^{\mathrm{TM}}\right.$, Thermo Fisher Scientific, Grand Island, NY, USA). First, the diluted siRNA was mixed with Lipofectamine for 10 $\mathrm{min}$ at room temperature. Then, the siRNA-lipid complex was added to the medium and was incubated for $48 \mathrm{~h}$ for subsequent experiments. The specific ON-TARGET plus $^{\text {TM }}$ SMARTpool EIF2S1 (eIF2 $\alpha$, L-015389), ATF4 (L-005125), SLC7A11 (xCT, L-007612), EIF2AK3 (PERK, L-004883), EIF2AK4 (GCN2, L-005314) and non-target (scramble, D-001810) siRNAs were used in these experiments.

\section{Kaplan-Meier plotter analysis}

This online database was based on the GEO (Affymetrix microarrays only), EGA and TCGA databases. The two patient cohorts were compared by KaplanMeier (KM) survival plots, and hazard ratios with $95 \%$ confidence intervals and log rank $p$ values were calculated using online software, as a previous study described [49]. In the gastric cancer database, the specific gene, $\mathrm{xCT}$ (SLC7A11, gene symbol Affy ID 209921_at), was used in this KM analysis. In the specific clinical cohorts receiving 5-FU based adjuvant therapy, a total of 153 patients were analyzed for overall survival (OS) and progression free survival (PFS) by KM analysis. 


\section{Detection of the level of intracellular ROS and mitochondrial ROS}

DCFH-dA and MitoSOX Red were used to determine the intracellular and mitochondrial ROS, respectively. After incubation with $5 \mu \mathrm{M}$ DCFH-dA for $30 \mathrm{~min}$ or $10 \mu \mathrm{M}$ MitoSOX Red for $10 \mathrm{~min}$, cells were washed with PBS, trypsinized, and re-suspended in PBS, as in previous methods. The DCF fluorescence intensity at FL1 and the MitoSOX Red fluorescence intensity at FL2 were determined by flow cytometry. A FACS Calibur flow cytometer (Becton Dickinson Bedford, MA, USA) equipped with a 488-nm argon laser was used for the flow cytometric analysis. The excitation wavelength was set at $488 \mathrm{~nm}$. In each measurement, a minimum of 15000 cells were analyzed. Data were acquired and analyzed using Cell Quest software (Becton Dickinson). The relative change in the mean fluorescence intensity was calculated as the ratio between the mean fluorescence intensity in the channel of the treated cells and that in the channel of the control cells.

\section{Human XCT promoter construction and reporter luciferase activity assay}

Cells $\left(2 \times 10^{5}\right)$ were seeded in 6-well culture plates and were transfected using Turbofect transfection reagent (Thermo Fisher Scientific), according to the manufacturer's instructions. Cells were co-transfected with $4 \mu \mathrm{g}$ of $\mathrm{xCT}$ reporter vector and $4 \mu \mathrm{g}$ of $\mathrm{pEGFP}-\mathrm{C} 2$ per well. The wild-type, ARE-mutant, and AARE-mutant $\mathrm{xCT}$ promoter constructs in the pGL3 luciferase reporter vector (Promega, Madison, WI, USA) are described in Supplementary Figure S1H. The human xCT promoters with ARE mutants or AARE mutants were created by site-direct mutagenesis, according to previous studies $[15,50,51]$. After transfection, cells were incubated in medium containing the indicated drugs or vehicle for $24 \mathrm{~h}$ and were collected for the determination of luciferase activity. The reporter assay was performed with the Promega luciferase assay system (Madison, WI, USA), following the manufacturer's manual. The green fluorescence intensity of pEGFP was used to normalize for transfection efficiency, and the relative ratio of luciferase to $\mathrm{pEGFP}$ was indicated as the reporter activity.

\section{Statistical analysis}

All of the data are presented as the mean \pm SEM. Sigmaplot software, version 10.0 (Systat Software), and GraphPad PRISM software, version 5 (GraphPad Software), were used for all statistical analyses. The statistical significance of the differences between two groups was evaluated using Student's t test. A $p$ value $<0.05$ was considered to be statistically significant.

\section{Abbreviations}

5-FU: 5-fluorouracil; ARE: antioxidant response element; AARE: amino acid response element; ATF4: activating transcription factor 4; ATP: adenosine triphosphate; BiP: immunoglobulin heavy chain-binding protein; BSA: bovine serum albumin; BSO: buthionine sulphoximine; CHOP: CEBP homologous; CisR: cisplatin-resistant; DCFHdA: 2'7'-dichlorodihydrofluorescein diacetate; DMEM: Dulbecco's modified Eagle's medium; DMSO: dimethyl sulfoxide; EDTA: ethylenediaminetetraacetic acid; eIF2 $\alpha$ : eukaryotic initiation factor $2 \alpha$; FBS: fetal bovine serum; GADD34: DNA damage-inducible protein 34; GADPH: glyceraldehyde 3-phosphate dehydrogenase; GCN2: general control nonderepressible-2; GSH: glutathione; HR: hazard ratio; HRI: heme-regulated inhibitor eIF2 kinase; MDR: multidrug resistance protein; MRP: multidrug resistance-associated protein; mtDNA: mitochondrial DNA; MTT: 3-(4,5-cimethylthiazol-2-yl)-2,5-diphenyl tetrazolium bromide; NAC: N-acetyl cysteine; NEAA: non-essential amino acids; Nrf2: nuclear factor erythroid 2-related factor-2; OS: overall survival; P/S: penicillin/ streptomycin; PCR: polymerase chain reaction; PERK: protein kinase R-like endoplasmic reticulum kinase; PFS: progression free survival; PKR: dsRNA-activated protein kinase R; PMSF: phenylmethanesulfonyl fluoride; PVDF: polyvinylidene difluoride; ROS: reactive oxygen species; RT: reverse transcription; SDS: sodium dodecyl sulfate; SRB: sulforhodamine B; SSA: sulfasalazine; TCA: trichloroacetic acid

\section{ACKNOWLEDGMENTS}

This work was supported by grants V103A-020, V104A-020 and V105A-018 from the Taipei Veterans General Hospital, Taipei, Taiwan, and partly by a grant from the Ministry of Education, Aim for the Top University Plan; grants MOST104-2320-B-010-031 and MOST 105-2320B-010-034 from the Ministry of Science and Technology, Taiwan; and MOHW105-TDU-13-211-134003 from the Ministry of Health and Welfare, Taiwan.

\section{CONFLICTS OF INTEREST}

The authors in this study declare no conflicts of interest.

\section{Authors' contributions}

S.F. Wang performed the experiments, analyzed the data, and wrote the manuscript. M.S. Chen participated in interpreting the results, established the $\mathrm{xCT}$ promoter luciferase reporter constructs, and corrected the manuscript. Y.C. Chou, Y.F. Ueng, P.H. Yin, and T.S. Yeh corrected the manuscript. H.C. Lee coordinated the study and wrote the manuscript. All of the authors read and approved the final manuscript. 


\section{REFERENCES}

1. Torre LA, Bray F, Siegel RL, Ferlay J, Lortet-Tieulent J and Jemal A. Global cancer statistics, 2012. CA Cancer J Clin. 2015; 65:87-108.

2. Wagner AD, Grothe W, Haerting J, Kleber G, Grothey A and Fleig WE. Chemotherapy in advanced gastric cancer: a systematic review and meta-analysis based on aggregate data. J Clin Oncol. 2006; 24:2903-2909.

3. Hanahan D and Weinberg RA. Hallmarks of cancer: the next generation. Cell. 2011; 144:646-674.

4. Lee HC and Wei YH. Mitochondrial role in life and death of the cell. J Biomed Sci. 2000; 7:2-15.

5. Wu CW, Yin PH, Hung WY, Li AF, Li SH, Chi CW, Wei $\mathrm{YH}$ and Lee HC. Mitochondrial DNA mutations and mitochondrial DNA depletion in gastric cancer. Genes Chromosomes Cancer. 2005; 44:19-28.

6. Hung WY, Wu CW, Yin PH, Chang CJ, Li AF, Chi CW, Wei YH and Lee HC. Somatic mutations in mitochondrial genome and their potential roles in the progression of human gastric cancer. Biochim Biophys Acta. 2010; 1800:264-270.

7. Quiros PM, Mottis A and Auwerx J. Mitonuclear communication in homeostasis and stress. Nat Rev Mol Cell Biol. 2016; 17:213-226.

8. Hsu CC, Tseng LM and Lee HC. Role of mitochondrial dysfunction in cancer progression. Exp Biol Med. 2016; 241:1281-1295.

9. Chang CJ, Yin PH, Yang DM, Wang CH, Hung WY, Chi $\mathrm{CW}$, Wei $\mathrm{YH}$ and Lee HC. Mitochondrial dysfunctioninduced amphiregulin upregulation mediates chemoresistance and cell migration in HepG2 cells. Cell Mol Life Sci. 2009; 66:1755-1765.

10. Hung WY, Huang KH, Wu CW, Chi CW, Kao HL, Li AF, Yin $\mathrm{PH}$ and Lee HC. Mitochondrial dysfunction promotes cell migration via reactive oxygen species-enhanced beta5integrin expression in human gastric cancer SC-M1 cells. Biochim Biophys Acta. 2012; 1820:1102-1110.

11. Siddik ZH. Cisplatin: mode of cytotoxic action and molecular basis of resistance. Oncogene. 2003; 22:7265-7279.

12. Lu SC. Glutathione synthesis. Biochim Biophys Acta. 2013; 1830:3143-3153.

13. Lo M, Wang YZ and Gout PW. The $\mathrm{x}(\mathrm{c})$ - cystine/glutamate antiporter: a potential target for therapy of cancer and other diseases. J Cell Physiol. 2008; 215:593-602.

14. Shih AY, Johnson DA, Wong G, Kraft AD, Jiang L, Erb H, Johnson JA and Murphy TH. Coordinate regulation of glutathione biosynthesis and release by Nrf2-expressing glia potently protects neurons from oxidative stress. J Neurosci. 2003; 23:3394-3406.

15. Sato H, Nomura S, Maebara K, Sato K, Tamba M and Bannai S. Transcriptional control of cystine/glutamate transporter gene by amino acid deprivation. Biochem Biophys Res Commun. 2004; 325:109-116.
16. Lewerenz $\mathrm{J}$ and Maher P. Basal levels of eIF2alpha phosphorylation determine cellular antioxidant status by regulating ATF4 and xCT expression. J Biol Chem. 2009; 284:1106-1115.

17. Wek RC, Jiang HY and Anthony TG. Coping with stress: eIF2 kinases and translational control. Biochem Soc Trans. 2006; 34:7-11.

18. Harding HP, Zhang Y, Zeng H, Novoa I, Lu PD, Calfon M, Sadri N, Yun C, Popko B, Paules R, Stojdl DF, Bell JC, Hettmann T, Leiden JM and Ron D. An integrated stress response regulates amino acid metabolism and resistance to oxidative stress. Mol Cell. 2003; 11:619-633.

19. Martinez-Reyes I, Sanchez-Arago $M$ and Cuezva JM. AMPK and GCN2-ATF4 signal the repression of mitochondria in colon cancer cells. Biochem J. 2012; 444:249-259.

20. Rouschop KM, Dubois LJ, Keulers TG, van den Beucken T, Lambin P, Bussink J, van der Kogel AJ, Koritzinsky M and Wouters BG. PERK/eIF2alpha signaling protects therapy resistant hypoxic cells through induction of glutathione synthesis and protection against ROS. Proc Natl Acad Sci U S A. 2013; 110:4622-4627.

21. Hsu CC, Wang $\mathrm{CH}$, Wu LC, Hsia CY, Chi CW, Yin $\mathrm{PH}$, Chang CJ, Sung MT, Wei YH, Lu SH and Lee HC. Mitochondrial dysfunction represses HIF-1alpha protein synthesis through AMPK activation in human hepatoma HepG2 cells. Biochim Biophys Acta. 2013; 1830:4743-4751.

22. Hsu CC, Wu LC, Hsia CY, Yin PH, Chi CW, Yeh TS and Lee HC. Energy metabolism determines the sensitivity of human hepatocellular carcinoma cells to mitochondrial inhibitors and biguanide drugs. Oncol Rep. 2015; 34:1620-1628.

23. Boyce M, Bryant KF, Jousse C, Long K, Harding HP, Scheuner D, Kaufman RJ, Ma D, Coen DM, Ron D and Yuan J. A selective inhibitor of eIF2alpha dephosphorylation protects cells from ER stress. Science. 2005; 307:935-939.

24. Park JS, Sharma LK, Li H, Xiang R, Holstein D, Wu J, Lechleiter J, Naylor SL, Deng JJ, Lu J and Bai Y. A heteroplasmic, not homoplasmic, mitochondrial DNA mutation promotes tumorigenesis via alteration in reactive oxygen species generation and apoptosis. Hum Mol Genet. 2009; 18:1578-1589.

25. Ye J, Kumanova M, Hart LS, Sloane K, Zhang H, De Panis DN, Bobrovnikova-Marjon E, Diehl JA, Ron D and Koumenis C. The GCN2-ATF4 pathway is critical for tumour cell survival and proliferation in response to nutrient deprivation. EMBO J. 2010; 29:2082-2096.

26. Singleton DC and Harris AL. Targeting the ATF4 pathway in cancer therapy. Expert Opin Ther Targets. 2012; 16:1189-1202.

27. Ma Y and Hendershot LM. Delineation of a negative feedback regulatory loop that controls protein translation during endoplasmic reticulum stress. J Biol Chem. 2003; 278:34864-34873. 
28. Silva JM, Wong A, Carelli V and Cortopassi GA. Inhibition of mitochondrial function induces an integrated stress response in oligodendroglia. Neurobiol Dis. 2009; 34:357-365.

29. Wek SA, Zhu S and Wek RC. The histidyl-tRNA synthetaserelated sequence in the eIF-2 alpha protein kinase GCN2 interacts with tRNA and is required for activation in response to starvation for different amino acids. Mol Cell Biol. 1995; 15:4497-4506.

30. Michel S, Canonne M, Arnould T and Renard P. Inhibition of mitochondrial genome expression triggers the activation of CHOP-10 by a cell signaling dependent on the integrated stress response but not the mitochondrial unfolded protein response. Mitochondrion. 2015; 21:58-68.

31. Mascarenhas C, Edwards-Ingram LC, Zeef L, Shenton D, Ashe MP and Grant CM. Gen4 is required for the response to peroxide stress in the yeast Saccharomyces cerevisiae. Mol Biol Cell. 2008; 19:2995-3007.

32. Berlanga JJ, Rivero D, Martin R, Herrero S, Moreno S and de Haro C. Role of mitogen-activated protein kinase Sty1 in regulation of eukaryotic initiation factor 2alpha kinases in response to environmental stress in Schizosaccharomyces pombe. PLoS Genet. 2010; 9:194-207.

33. Baker BM, Nargund AM, Sun T and Haynes CM. Protective coupling of mitochondrial function and protein synthesis via the eIF2alpha kinase GCN-2. PLoS genetics. 2012; 8:e1002760.

34. Padyana AK, Qiu H, Roll-Mecak A, Hinnebusch AG and Burley SK. Structural basis for autoinhibition and mutational activation of eukaryotic initiation factor 2alpha protein kinase GCN2. J Biol Chem. 2005; 280:29289-29299.

35. TTonigold M, Rossmann A, Meinold M, Bette M, Marken M, Henkenius K, Bretz AC, Giel G, Cai C, Rodepeter FR, Benes V, Grenman R, Carey TE, et al. A cisplatin-resistant head and neck cancer cell line with cytoplasmic p53(mut) exhibits ATP-binding cassette transporter upregulation and high glutathione levels. J Cancer Res Clin Oncol. 2014; 140:1689-1704.

36. Harris IS, Treloar AE, Inoue S, Sasaki M, Gorrini C, Lee $\mathrm{KC}$, Yung KY, Brenner D, Knobbe-Thomsen CB, Cox MA, Elia A, Berger T, Cescon DW, et al. Glutathione and thioredoxin antioxidant pathways synergize to drive cancer initiation and progression. Cancer Cell. 2015; 27:211-222.

37. Timmerman LA, Holton T, Yuneva M, Louie RJ, Padro M, Daemen A, Hu M, Chan DA, Ethier SP, van 't Veer LJ, Polyak K, McCormick F and Gray JW. Glutamine sensitivity analysis identifies the $\mathrm{xCT}$ antiporter as a common triple-negative breast tumor therapeutic target. Cancer Cell. 2013; 24:450-465.

38. Lanzardo S, Conti L, Rooke R, Ruiu R, Accart N, Bolli E, Arigoni M, Macagno M, Barrera G, Pizzimenti $\mathrm{S}$, Aurisicchio L, Calogero RA and Cavallo F. Immunotargeting of Antigen xCT Attenuates Stem-like Cell Behavior and Metastatic Progression in Breast Cancer. Cancer Res. 2016; 76:62-72.
39. Ishimoto T, Nagano O, Yae T, Tamada M, Motohara T, Oshima H, Oshima M, Ikeda T, Asaba R, Yagi H, Masuko $\mathrm{T}$, Shimizu T, Ishikawa T, et al. CD44 variant regulates redox status in cancer cells by stabilizing the $\mathrm{xCT}$ subunit of system xc(-) and thereby promotes tumor growth. Cancer Cell. 2011; 19:387-400.

40. Wada T, Ishimoto T, Seishima R, Tsuchihashi K, Yoshikawa M, Oshima H, Oshima M, Masuko T, Wright NA, Furuhashi S, Hirashima K, Baba H, Kitagawa Y, Saya H and Nagano O. Functional role of CD44v-xCT system in the development of spasmolytic polypeptide-expressing metaplasia. Cancer Sci. 2013; 104:1323-1329.

41. Kim NK, Park YS, Heo DS, Suh C, Kim SY, Park KC, Kang YK, Shin DB, Kim HT, Kim HJ and et al. A phase III randomized study of 5-fluorouracil and cisplatin versus 5-fluorouracil, doxorubicin, and mitomycin $\mathrm{C}$ versus 5-fluorouracil alone in the treatment of advanced gastric cancer. Cancer. 1993; 71:3813-3818.

42. Lorenzen S, Duyster J, Lersch C, von Delius S, Hennig M, Bredenkamp R, Peschel C and Lordick F. Capecitabine plus docetaxel every 3 weeks in first- and second-line metastatic oesophageal cancer: final results of a phase II trial. Br J Cancer. 2005; 92:2129-2133.

43. Lee JL, Ryu MH, Chang HM, Kim TW, Yook JH, Oh ST, Kim BS, Kim M, Chun YJ, Lee JS and Kang YK. A phase II study of docetaxel as salvage chemotherapy in advanced gastric cancer after failure of fluoropyrimidine and platinum combination chemotherapy. Cancer Chemother Pharmacol. 2008; 61:631-637.

44. Huang Y, Dai Z, Barbacioru C and Sadee W. Cystineglutamate transporter SLC7A11 in cancer chemosensitivity and chemoresistance. Cancer Res. 2005; 65:7446-7454.

45. Yoshikawa M, Tsuchihashi K, Ishimoto T, Yae T, Motohara T, Sugihara E, Onishi N, Masuko T, Yoshizawa K, Kawashiri S, Mukai M, Asoda S, Kawana H, Nakagawa T, Saya H and Nagano O. xCT inhibition depletes CD44vexpressing tumor cells that are resistant to EGFR-targeted therapy in head and neck squamous cell carcinoma. Cancer Res. 2013; 73:1855-1866.

46. Burgess DJ. Anticancer Drugs: Selective oxycution? Nat Rev Drug Discov. 2011; 10:658.

47. Dixon SJ, Lemberg KM, Lamprecht MR, Skouta R, Zaitsev EM, Gleason CE, Patel DN, Bauer AJ, Cantley AM, Yang WS, Morrison B, 3rd and Stockwell BR. Ferroptosis: an iron-dependent form of nonapoptotic cell death. Cell. 2012; 149:1060-1072.

48. Axten JM, Medina JR, Feng Y, Shu A, Romeril SP, Grant SW, Li WH, Heerding DA, Minthorn E, Mencken T, Atkins C, Liu Q, Rabindran S, et al. Discovery of 7-methyl-5-(1$\{[3$-(trifluoromethyl)phenyl]acetyl $\}$-2,3-dihydro-1H-indol5-yl)-7H-p yrrolo[2,3-d]pyrimidin-4-amine (GSK2606414), a potent and selective first-in-class inhibitor of protein kinase R (PKR)-like endoplasmic reticulum kinase (PERK). J Med Chem. 2012; 55:7193-7207. 
49. Gyorffy B, Surowiak P, Budczies J and Lanczky A. Online survival analysis software to assess the prognostic value of biomarkers using transcriptomic data in non-small-cell lung cancer. PLoS One. 2013; 8:e82241.

50. Ye P, Mimura J, Okada T, Sato H, Liu T, Maruyama A, Ohyama C and Itoh K. Nrf2- and ATF4-dependent upregulation of $\mathrm{xCT}$ modulates the sensitivity of $\mathrm{T} 24$ bladder carcinoma cells to proteasome inhibition. Mol Cell Biol. 2014; 34:3421-3434.

51. Sasaki H, Sato H, Kuriyama-Matsumura K, Sato K, Maebara K, Wang H, Tamba M, Itoh K, Yamamoto M and Bannai S. Electrophile response element-mediated induction of the cystine/glutamate exchange transporter gene expression. J Biol Chem. 2002; 277:44765-44771. 